
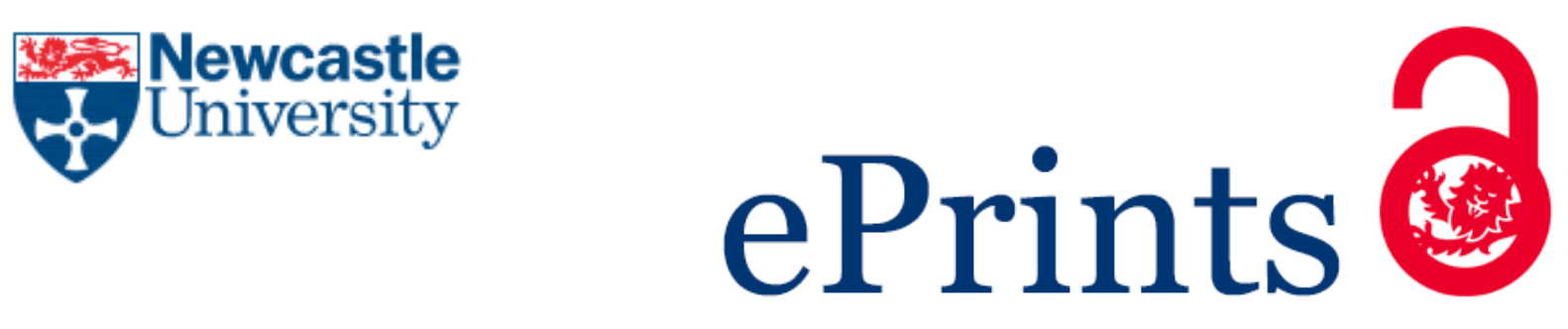

Onat A, Voltr P, Lata M.

An unscented Kalman filter-based rolling radius estimation methodology for railway vehicles with traction.

Proceedings of the Institution of Mechanical Engineers, Part F: Journal of Rail and Rapid Transit 2017

DOI: https://doi.org/10.1177/0954409717745201

Copyright:

(C) 2017 (IMechE 2017). Reprinted by permission of SAGE Publications

DOI link to article:

https://doi.org/10.1177/0954409717745201

Date deposited:

$15 / 02 / 2018$

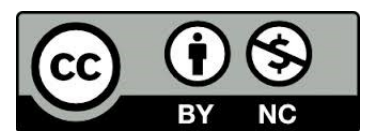

This work is licensed under a Creative Commons Attribution-NonCommercial 3.0 Unported License 


\title{
An Unscented Kalman Filter Based Rolling Radius Estimation Methodology for Railway Vehicles with Traction
}

\author{
Altan Onat ${ }^{1}$, Petr Voltr ${ }^{2}$ and Michael Lata ${ }^{1}$
}

\begin{abstract}
Condition monitoring for railway vehicle systems plays an important role to maintain safety and performance of railway vehicles. Rolling radius is one of the properties that should be monitored continuously for a predictive maintenance of a railway vehicle since it changes with time due to wheel wear. In this study, a model based condition monitoring methodology, which is based on unscented Kalman filter, is proposed. Model includes the torsional dynamics of an independently rotating tram wheel with a traction motor and a contact model. Rolling radius is estimated by considering the traction effort of the motor and angular velocity measurements. Proposed methodology is tested on a tram wheel test stand (roller-rig) which has a wheel on roller configuration. Firstly, mathematical model is validated by the measurements taken from the test stand. Secondly, unscented Kalman filter is applied as a parameter estimator. Results demonstrate that proposed scheme is promising to use for predictive condition monitoring of wheel profile for traction vehicles.
\end{abstract}

\section{Keywords}

rolling radius estimation, parameter estimation, unscented Kalman filter, model based condition monitoring, tram wheel, roller-rig, torsional dynamics

\section{Introduction}

A recent review article reported by $\mathrm{Li}$ et al. ${ }^{1}$ summarizes the existing approaches applied for railway vehicle on-board health (i.e. condition) monitoring systems. It is indicated that the focus of condition monitoring systems should be wheelrail health monitoring, suspension health monitoring, vehicle component health monitoring and running state monitoring. ${ }^{1}$ It is also stated by $\mathrm{Li}$ et al. ${ }^{1}$ that unscented Kalman filter, which is also used hereby, is generally considered to estimate wheel-rail friction coefficients in the railway industry. ${ }^{2 ; 3}$

Wheel profile condition monitoring methodologies can be classified into two group and these are methodologies based on vehicle mounted sensors and track-side sensors. ${ }^{1}$ Methodologies based on track-side sensors have the drawback of providing information only on the specific points on the track. Therefore, methodologies based on vehicle mounted sensors are preferable since they provide continuous information along the track.

One of the very first model based estimation schemes for wheel-rail profile estimation is given by Charles et al. ${ }^{4}$, and a lateral dynamic model of a wheelset is used. Measurements are taken from a generic wheelset model to estimate the conicity, which is a linearised parameter including secant gradient of rolling radius difference, and only a simulation study is presented without experimental validation. ${ }^{4} \mathrm{~A}$ state of art application by using time frequency techniques for detecting wheel flat and rail defects is presented by Liang et al. $^{5}$ and results are experimentally validated, whereas good agreement was achieved between simulated and measured acceleration results just for low wheel speed $(3.5 \mathrm{~km} / \mathrm{h})$. For high wheel speed, difference between simulations and measurements becomes higher and causes degradation in detection performance. ${ }^{5}$ Similar method, which also considers vertical dynamics of a vehicle and Fourier transform, is reported by Real et al. ${ }^{6}$ and the focus is detecting the defects on rails. A recent article that reveals direct detection methods for wheel defects is given by Alemi et al. ${ }^{7}$

On-track experiments for railway vehicles is burdensome and time consuming. In order to overcome this issue, rollerrig test stands are used for research purposes to enhance the performance of vehicles. Adhesion, wheel-rail contact and traction have been attracted attention of many researchers since the first days of railway vehicles and roller-rigs are extensively used for a better observation of these phenomena. According to the research purpose, the substitution of the rail with the roller and the vehicle with the substitutive configuration differ. The details of the several cases for substitution and the differences from real case are reported by Voltr ${ }^{8}$, Liu and Bruni ${ }^{9}$.

Reported roller-rig configurations in literature differ in size. ${ }^{10 ; 11}$ According to the size, roller-rigs can be specified as full scale and reduced scale. Full scale test stands are generally useful to understand behaviour of complete railway vehicle systems, whereas scaled roller-rigs with

\footnotetext{
${ }^{1}$ Department of Transport Means and Diagnostics, Faculty of Transport Engineering, University of Pardubice, Czechia

${ }^{2}$ Educational and Research Centre in Transport, Faculty of Transport Engineering, University of Pardubice, Czechia
}

\section{Corresponding author:}

Altan Onat, Department of Transport Means and Diagnostics, Faculty of Transport Engineering, University of Pardubice, Czechia

Email: altanonat@anadolu.edu.tr 
different configurations are the cheap, easy to construct and purposeful version of the full scale ones. Although many improvements have been carried out for roller-rigs to replace them with real vehicles, more research is still needed, especially by considering a multidisciplinary engineering perspective.

For many years, experimental research has been conducted in Faculty of Transport Engineering, Pardubice, Czechia on a tram wheel test stand originally built by VÚKV (Výzkumný Ústav Kolejových Vozidel - Rail Vehicle Research Institute). Extensive reconstruction for this test stand has been made at the Faculty of Transport Engineering. Adhesion ${ }^{12-14}$ and traction $^{15 ; 16}$ are the focus of the research by using this tram wheel test stand. It is a full scale roller-rig having a wheel on roller configuration. This wheel on roller configuration has some advantages and disadvantages. ${ }^{17}$ Most significant advantage of such configuration is that it provides better contact mechanics (adhesion, contact geometry, creep forces, etc.) studies. The most obvious disadvantage is that it does not allow to study hunting and curving dynamics.

Especially on such roller-rigs, which have wheel on roller configuration, the effect of adhesion conditions is studied extensively. ${ }^{18 ; 19}$ In order to simulate such adhesion conditions water, oil, sand, lubricants etc. ${ }^{18 ; 19}$ are used. Use of such contaminants causes wear and defects on wheel and roller surface. This situation degrades the accuracy in such studies as contact is directly formed by the profiles. Therefore, measuring profiles and rolling radii is crucial during experiments. For the case in real vehicles, such wear and defects can even cause safety problems.

In this study, a model based condition monitoring scheme is proposed to estimate rolling radius of an independently rotating tram wheel and the effectiveness of the methodology is proven with experiments carried out on a tram wheel test stand. Firstly, this methodology provides continuous inspection of the rolling radius of an independently rotating tram wheel. This inspection is directly related with the wheel wear and defects. Therefore, such wear and defects can easily be identified by this estimation methodology. Besides, this condition monitoring scheme is effective for higher translational speeds (e.g. $30 \mathrm{~km} / \mathrm{h}$ ) than the previously reported experimental and signal based scheme for wheel defect detection. ${ }^{5}$ Furthermore, signal based schemes (e.g. Liang et al. $^{5}$ ) for wheel profile monitoring just indicates the existence of a wheel wear and/or defect and they do not provide the severity or the magnitude of this wear and/or defect. Methodology presented hereby shows that with certain assumptions magnitude of the rolling radius, which is directly related with wheel wear and defects, can be monitored. As a second significance, this scheme combines the information from a mathematical model describing the physical system and the measurements taken from the test stand (or a vehicle). Therefore, in case of model uncertainties or a sensory fault, such a model based methodology provides more accurate state estimates. Such situation is especially apparent in traction control systems. Mathematical models, which are similar to the model considered hereby, are used for traction control systems. ${ }^{20}$ Nevertheless, model or measurement uncertainties are not taken into account. Therefore, this methodology can be used as an auxiliary system to improve effectiveness and performance of traction and adhesion control systems.

In this case of application, both wheel and roller are driven and angular velocity measurements are taken from both wheel and roller. In practice for railway vehicles, there are rails instead of roller. Therefore, angular velocity measurement assumption of roller fails in practice. However, in this application, angular velocity measurement on a rollerrig is necessary to obtain creep value, so that creep forces at contact can be described accurately. In order to obtain creep forces and overcome this issue, the translational velocity of the vehicle should be measured accurately and this can be achieved by modern and accurate velocity measurement systems (e.g. GPS - Global Positioning System etc.). Thus, instead of angular velocity of the roller, translational velocity of the railway vehicle is taken as measurement in practice. This scheme provides good results in case of necessary excitation (i.e. torque). Another drawback of this application is when there is lack of excitation (i.e. torque) in the system results of this scheme is unreliable as it is in any parameter estimation scheme. Application of this methodology is not straightforward for wagons without traction motors and in steady rolling case (i.e. without torque) of locomotives. For these cases, this methodology can be applied with different models which continuous excitation (e.g. track irregularities) can be considered. This is examined in details in the discussion section of the text.

An illustration which summarizes the proposed methodology can be found in Figure 1. In this study, in order to show effectiveness of the methodology, it is applied to the mentioned tram wheel test stand. Therefore, firstly, an analysis of the mentioned tram wheel stand is introduced and a model to represent this physical system is proposed. This analysis includes derivation of a contact model, inspection of the electric motors and torsional dynamics of the stand. After the model is validated in comparison with measurements, the rolling radius estimation scheme is presented. Lastly, the use of such estimation scheme for real vehicles is discussed.

\section{Tram wheel test stand}

The test rig comprises a full-scale tramway wheel and a roller ("rotating rail") manufactured from a railway wagon wheel. Both rolling components are carried by an upright frame, the former attached to a swinging arm with a pneumatic spring for normal force, the latter mounted in bearings of a base plate. The roller assembly can be set into the angle of attack and, to a limited extent, shifted laterally. To provide traction or braking at the tram wheel, the machine is equipped with a torque-controlled permanent magnet synchronous motor (i.e. PMSM). Its effect is opposed by an asynchronous motor of the roller which is operated to keep constant speed. ${ }^{14} \mathrm{~A}$ diagram and the photos of the test stand can be seen in Figure 2 and Figure 3, respectively.

The normal force is provided by a pressurised air spring and the air pressure is observed by a pressure transmitter of type DMP331 produced by BD SENSORS, Germany. The incremental rotary encoders of type IRC 315 produced by LARM, Czechia mounted on both shafts provide the angular velocities of the wheel $\left(\omega_{w}\right)$ and the rail roller $\left(\omega_{r}\right)$. This type of encoder has physically 4096 pulses per revolution 


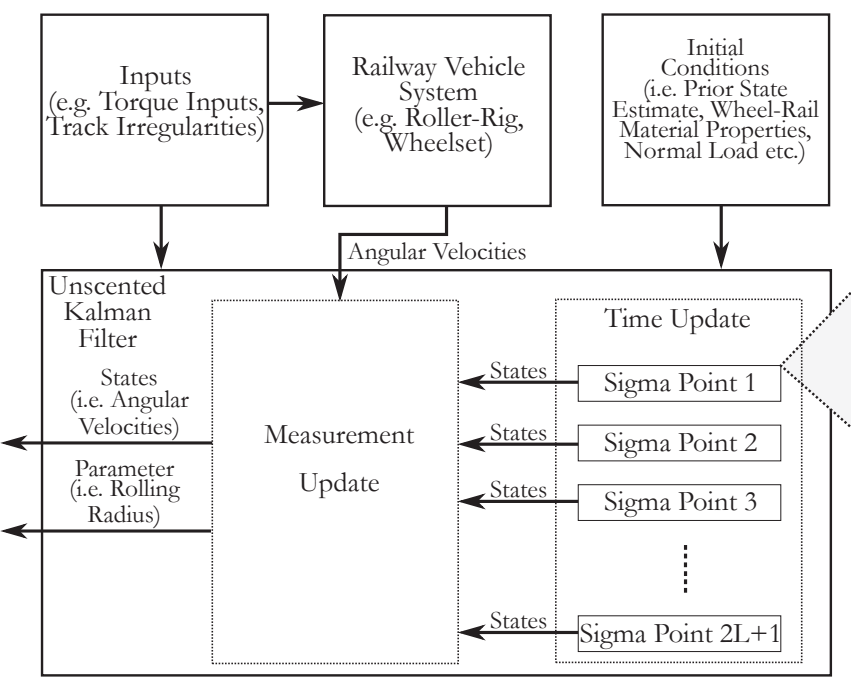

Figure 1. Structure of the methodology

(i.e. PPR), but by considering X4 mode of evaluation 16384 PPR are obtained. The tangential force $T$ and the coefficient of adhesion $\mu$ (ratio of the tangential force to the normal force) are calculated from the output of a torque transducer on the roller shaft. In a recent study ${ }^{21}$, which is carried out on the same test stand, it is indicated that based on the torque calibration data the uncertainty of the adhesion value is approximately 0.02 . All sensors were connected to the DAQ device of type NI USB-6341 produced by National Instruments, United States which has 500000 samples per second for all channels in total. However, this is not used to the maximum and the sampling rate used in measurements is normally $200 \mathrm{~Hz}$. The A/D converter used in measurements has 16 bit resolution. Similar configuration for contact force calculation is presented by using a dynamometer by Meymand et al. ${ }^{22}$. The electric motor connected to the wheel is a PMSM, which has a nominal power of $58 \mathrm{~kW}$, a nominal torque of $852 \mathrm{Nm}$ and maximum torque of 2000 $\mathrm{Nm}$. Additionally, the electric motor connected to the roller is an asynchronous motor produced by the manufacturer MEZ Brno, Czechia which has a nominal power of $55 \mathrm{~kW}$ and a nominal torque of $891 \mathrm{Nm}$. The nominal rolling diameter of the wheel is $700 \mathrm{~mm}$ and the nominal rolling diameter of the roller is $905 \mathrm{~mm}$.

The roller rig simulates the driving system of some trams used in Czechia. These trams include independently rotating wheels. The motors are carried by the bogie frame in such way that it may tilt about the longitudinal axis. This configuration compensates vertical movements in primary suspension in cooperation with a constant velocity (i.e. cv) joint at the other end of the drive shaft. Characterisation of the test stand can be further used to propose high performance traction control systems, new wheel profiles and suspension systems for high adhesion and low wear during the tram design process.

\section{Electrical motors attached to wheel and roller}

As mentioned previously, the tram wheel is driven by a PMSM with feedback flux weakening control. ${ }^{16}$ The details of this motor and control procedure is presented by Doleček et al. ${ }^{16}$, so hereby details about the motor and control method are omitted. In this study, torque applied by this motor is provided as a torque request from this controller. However, there was no attention paid previously to the asynchronous motor attached to the roller which is used for braking. In order to obtain opposing torque of this asynchronous motor with respect to angular velocity, its equivalent circuit model should be obtained by some simple tests. ${ }^{23}$ These are no load test, blocked rotor test and measuring per phase stator resistances. After these tests are conducted, the Thevenin equivalent circuit is obtained at $50 \mathrm{~Hz}$ and the equivalent circuit for this frequency can be seen in Figure 4. This asynchronous motor is controlled with an open loop V/f (i.e. volts per Hertz) control scheme by using a rectifier and inverter couple as it is seen in the diagram given in Figure 5. When the frequency decreases, in order to keep constant flux and to protect the motor, line voltage must also decrease proportionally to frequency so that V/f ratio can be kept constant. ${ }^{23}$ Mechanical torque of the asynchronous motor can be given as follows:

$$
T_{\text {mech }}=\frac{3}{\omega_{\text {syn }}} \frac{V_{T h}^{2}}{\left(R_{T h}+\frac{R_{2}^{\prime}}{s}\right)^{2}+\left(X_{T h}+X_{2}^{\prime}\right)^{2}} \frac{R_{2}^{\prime}}{s},
$$

where $\omega_{\text {syn }}$ is the synchronous angular speed of the motor and $s$ is the slip. The slip is simply the difference between the rotor speed and synchronous speed. ${ }^{23}$

\section{Contact analysis}

\section{Geometrical problem}

After extensive use, the wheel has recently been reprofiled, using the profiles "W169" and "R169". These profiles have been designed particularly for the roller-rig, and their conicity and curvatures are similar to those of common wheels and rails used in the tramway network of Prague, Czechia. Wheel is based on the theoretical profile, named "VM" and designed by VÚKV. The roller is also reprofiled by considering the theoretical profile of the rail, named "NT1" and designed by the manufacturer Trrinecké 


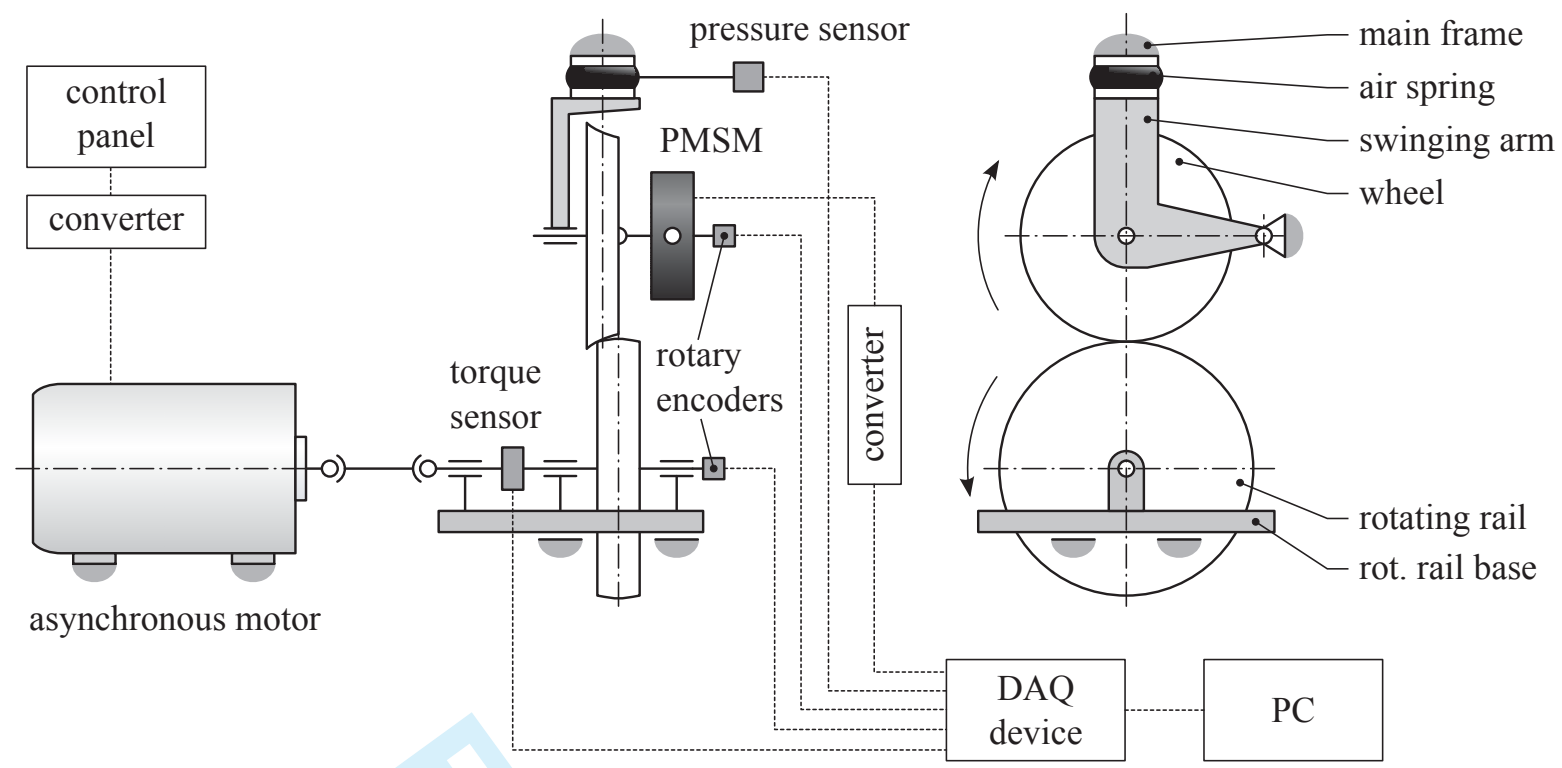

Figure 2. Diagram of the test stand

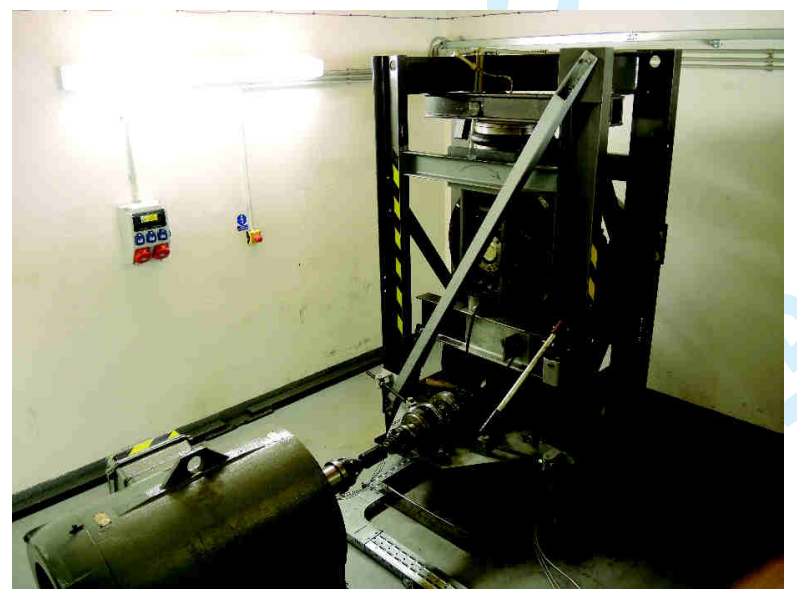

(a)

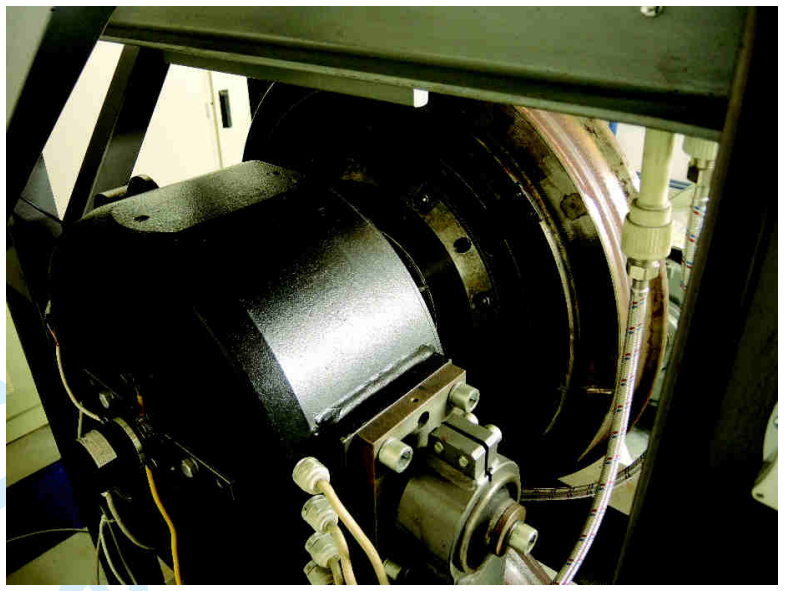

(b)

Figure 3. The tram wheel test rig in the laboratory of the University of Pardubice

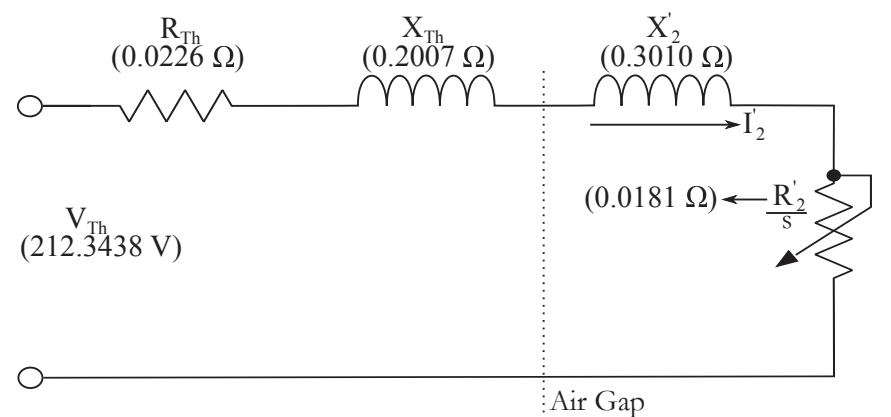

Figure 4. Thevenin equivalent circuit of the Asynchronous motor

Železárny, Czechia. Measured wheel/roller profiles by using a miniprof device are illustrated in Figure 6.

In order to analyse the contact, rolling radii in longitudinal (roller diameter - $0.9043 \mathrm{~m}$, wheel diameter - $0.6964 \mathrm{~m}$ ) and lateral directions and the contact angle must be obtained. The longitudinal rolling radii for the wheel/roller is obtained by using measurements by a diameter tape. Mathematical tools

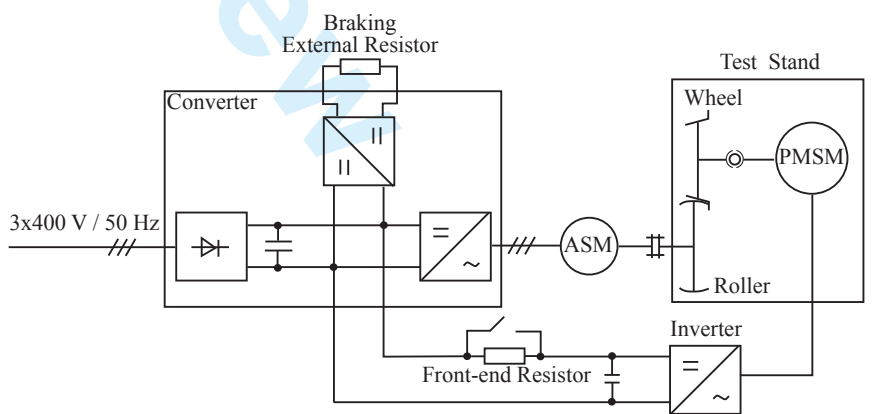

Figure 5. Diagram for the electrical layout ${ }^{16}$

are used to obtain the lateral rolling radii and the contact angle. The conicity angle of the wheel is defined as follows:

$$
\tan \delta_{w}=\frac{d z_{w}}{d y_{w}}
$$

and the signed lateral curvature is 


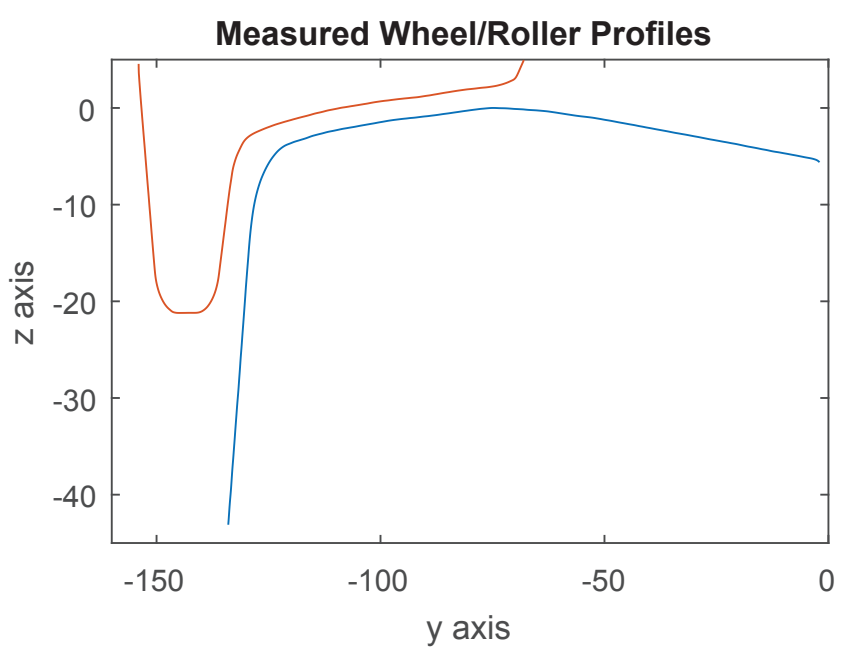

Figure 6. Measured profiles

$$
\kappa=\frac{\frac{d^{2} z_{w}}{d y_{w}^{2}}}{\left(1+\left(\frac{d z_{w}}{d y_{w}}\right)^{2}\right)^{\frac{3}{2}}}
$$

Obtained results can be seen for the conicity angle of the wheel in Figure 7 and for lateral curvatures of the wheel/roller in Figure 8. A simple second order differentiation is used to take derivatives indicated in Equations 2, 3. The results are unsuitable to use them in a Hertzian contact analysis due to sudden changes of curvature values. Therefore, a simple moving average filter with a window length of $5 \mathrm{~mm}$ is used. It should be mentioned that reference point (i.e. origin) in the y-axis of the Figures 7, 8 and 9 are same as the origin of the Figure 6.

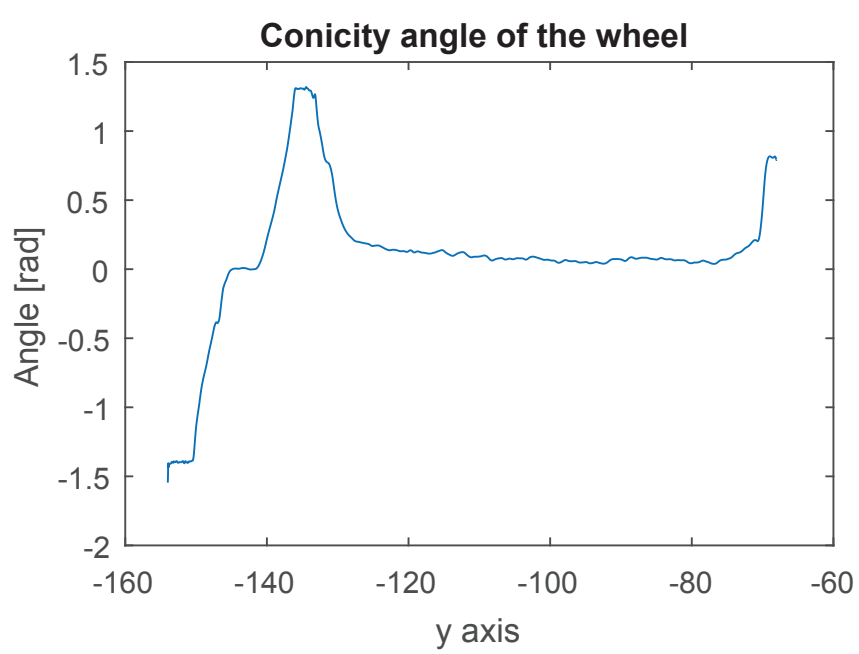

Figure 7. Conicity angle of the wheel with respect to y axis

A contact point must be determined to obtain a contact patch. In order to find this contact point rigid and quasielastic ${ }^{24 ; 25}$ contact search methods are applied. The quasielastic contact search method is a more realistic approach in comparison with the rigid method and it has less computational complexity than the finite element analysis. Simply, the rigid search is based on finding the minimum of distance on the $\mathrm{z}$ axis, whereas the quasi-elastic method is based on finding the contact locus by using a weighted average function. A similar application of the quasi-elastic contact search method for a roller-rig system also presented by Heckmann et al. ${ }^{26}$. An illustration to show the difference between the two methods is given in Figure 9. In this study, the result of the quasi-elastic contact search is considered. The contact angle is found as 0.0503 radians, the lateral curvatures of wheel and roller are found as $3.4306 \mathrm{~m}^{-1}$ and $0.5259 \mathrm{~m}^{-1}$, respectively. It is observed that the results are also consistent with the curvatures obtained by using theoretical profiles.

\section{Normal Problem}

In order to find the contact patch, theory of Hertz ${ }^{27}$ is used. Details of the solution methodology for the normal problem, which is considered in this study, are reported by Onat et al. ${ }^{28}$ For a $13 \mathrm{kN}$ normal load applied by the air spring, the Hertzian contact ellipse has the semi-length of $2.605 \mathrm{~mm}$ and the semi-width of $2.622 \mathrm{~mm}$. This result is consistent with the finite element and analytical result reported in a previous study. ${ }^{29}$ Moreover, after the Hertzian contact is obtained, a virtual penetration method, which is proposed by Kik and Piotorowski ${ }^{30}$ is used. This method is based on the correction of the shape by considering the virtual penetration value which is equal to 0.55 times the penetration value calculated by using the theory of Hertz. The new contact shape is given by

$$
a=\sqrt{\frac{0.55 \delta_{0}}{A}}, b=\sqrt{\frac{0.55 \delta_{0}}{B}},
$$

where $a, b$ are the contact patch ellipse length and width, and $A, B$ are the Hertzian curvatures. By using this method, the result of this study is more consistent with the carbon test result presented in a previous study ${ }^{29}$ in which the semilength of $2.5 \mathrm{~mm}$ and the semi-width of $3 \mathrm{~mm}$ are found, whereas it is found here as $2.674 \mathrm{~mm}$ and $3.028 \mathrm{~mm}$ for the same normal load after the shape correction, respectively .

\section{Friction model}

During measurements dry surface conditions are considered. The variable friction model, which is presented by Polach ${ }^{31}$, is used. In this model, adhesion is dependent on the slip velocity. The slip velocity depends on the dimensionless creep and the translational velocity of the wheel. The dimensionless creep and the creep due to spin $(1 / m)$ are calculated as

$$
\begin{aligned}
& s_{x}=\frac{v_{r}-v_{w}}{v_{r}}, \\
& s_{\phi}=\frac{\sin \delta_{w}}{r_{e q_{x}}},
\end{aligned}
$$

where $v_{w}$ and $v_{r}$ are the translational velocities of the wheel and roller, respectively and $\delta_{w}$ is the contact angle. Unlike the real wheel/rail case, in this scheme roller also rotates. Therefore, in order to calculate the spin creepage, an equivalent radius $r_{e q_{x}}$ should be considered and can be given as:

$$
\frac{1}{r_{e q_{x}}}=\frac{1}{r_{w_{x}}}+\frac{1}{r_{r_{x}}},
$$




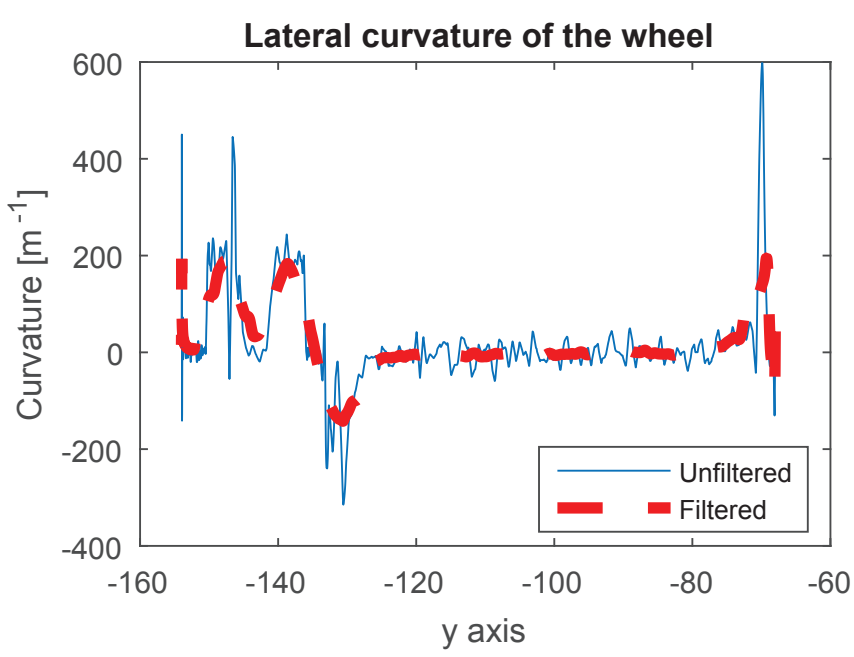

(a)

Figure 8. Lateral curvatures

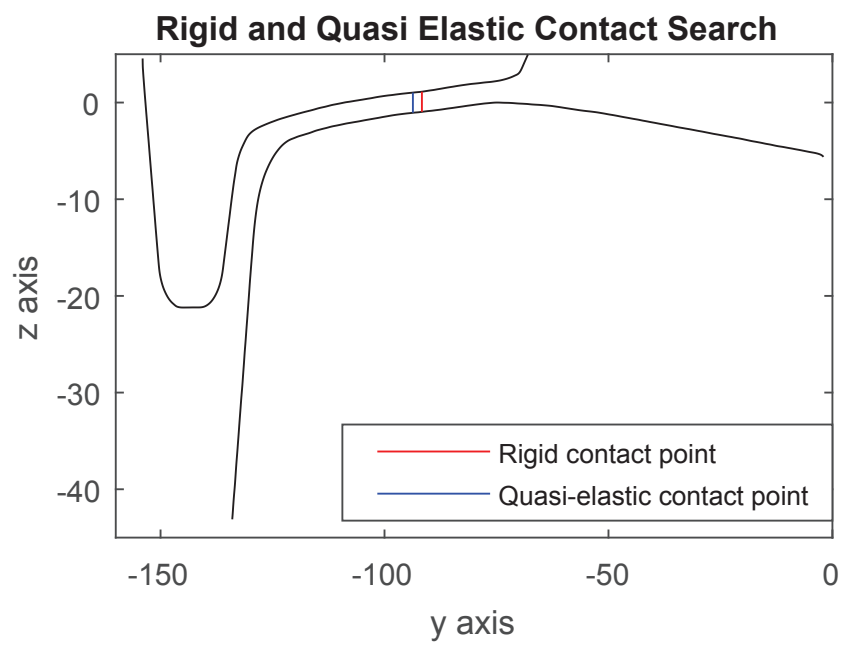

Figure 9. Contact search

where $r_{w_{x}}$ and $r_{r_{x}}$ are the rolling radii of the wheel and roller, respectively. The slip velocity simply equals to $w=$ $s_{x} \times v_{w}$ and friction coefficient can be given as:

$$
\mu=\mu_{0}\left[(1-A) e^{-B w}+A\right],
$$

where $\mu_{0}$ is the maximum friciton coefficient, $w[\mathrm{~m} / \mathrm{s}]$ is the magnitude of the slip velocity, $B[\mathrm{~s} / \mathrm{m}]$ is the coefficient of exponential decrease and $A$ is the ratio of limit friction coefficient $\mu_{\infty}$ to $\mu_{0}$. Here, by using previous knowledge from experimental study for dry conditions, $\mu_{0}, A, B$ is selected as 0.4, 0.4 and 0.6, respectively. Comparison of the model with respect to the adhesion measurement can be found in Figure 10. It should be indicated that adhesion measurements are done by using the torque transducer which is connected to the shaft of the roller. Torque value, which is provided by the transducer, is also used for determination of the creep force as measurement. Shortly, determination of creep force leads to the adhesion coefficient considering the normal force measurement by using pressure transmitter. In this study, dry surface conditions are considered, but a creep force model proposed for water induced low adhesion, which

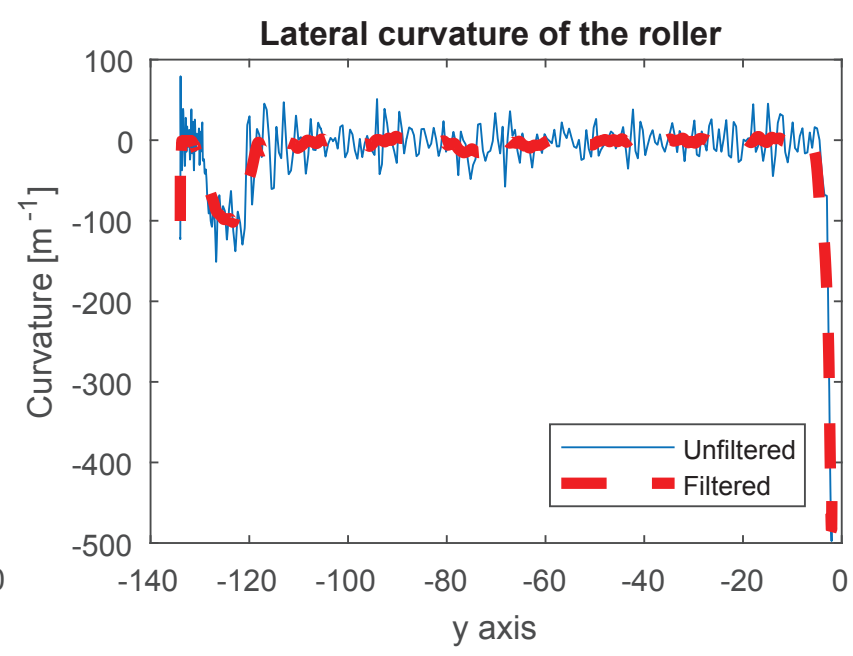

(b)

experiments are carried out on the same test stand, can be found in the study by Trummer et al. ${ }^{21}$

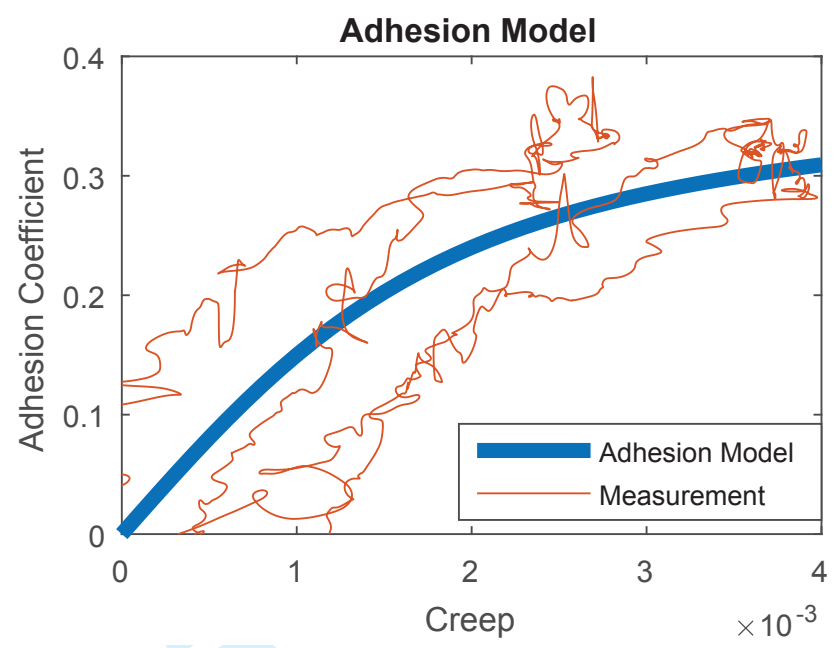

Figure 10. Adhesion model comparison

\section{Tangential problem}

The method namely Fastsim, which is proposed by Kalker ${ }^{32}$ and used in many multibody simulation software, is considered for the solution of tangential problem. Fastsim is based on the simplified theory proposed by Kalker and this theory is built upon the fact that

$$
\mathbf{u}=L \mathbf{p},
$$

where $\mathbf{u}=\left[\begin{array}{lll}u_{x} & u_{y} & u_{z}\end{array}\right]$ is the deformation vector, $\mathbf{p}=$ $\left[\begin{array}{lll}\tau_{x} & \tau_{y} & p_{z}\end{array}\right]$ is the traction vector and $L$ is the flexibility parameter. Flexibilities for the longitudinal direction and spin are given as follows:

$$
L_{x}=\frac{8 a}{3 G c_{11}}, L_{\phi}=\frac{\pi a^{2}}{4 G c_{23} \sqrt{a b}}
$$

where $G$ is the shear modulus of rigidity $\left(8 \times 10^{10} \mathrm{~Pa}\right)$, $c_{11}$ and $c_{23}$ are the coefficients from Kalker's linear theory. 


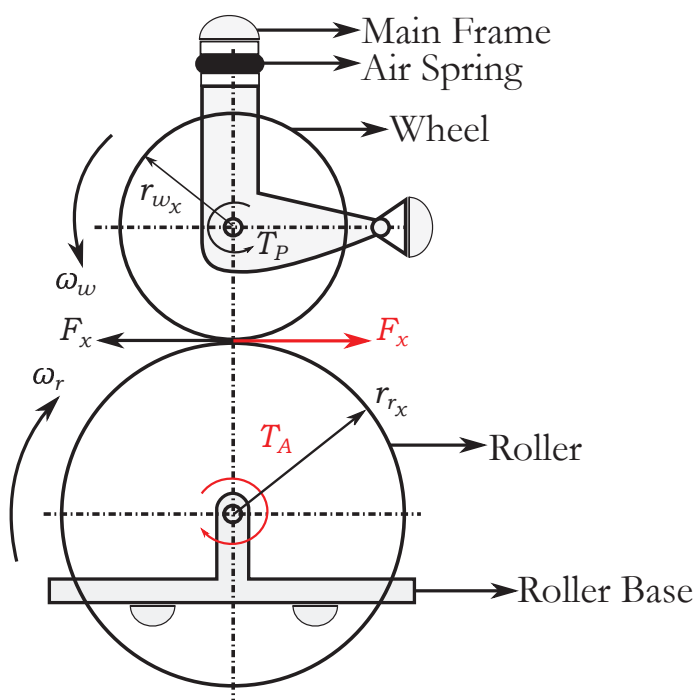

Figure 11. Forces and torques acting on roller-rig

Polynomial approximation for these coefficients are given in Appendix A. In this case of application, since there is no lateral creepage, the lateral flexibility is not given in Equation 9. For derivations and proofs, readers are reffered to read the study reported by Kalker ${ }^{32}$.

Furthermore, polynomial approximation for Kalker coefficients, which are given in Appendix A, includes the Poisson's ratio as a variable different from the polynomial approximations reported previously which assumes the Poisson's ratio of the steel is equal to the $0.27 .{ }^{10}$ The polynomial approximation given in Appendix A is especially useful when a study is carried out for a roller-rig test stand or vehicle system which includes wheel and roller (or rail) with different steels. Similar approximation, which also considers Poisson's ratio as a variable, is reported by Meymand ${ }^{17}$ and also different fit models are compared. It is shown by Meymand ${ }^{17}$ that polynomial approximation for fit provides the least root mean squared error.

\section{Torsional dynamic model}

A simple torsional dynamic model is considered. Forces and torques, which are affecting the roller-rig, can be seen in Figure 11.

Torsional dynamic model of the roller-rig can be expressed as follows:

$$
\begin{array}{r}
\frac{d \dot{\omega}_{r}}{d t}=\frac{T_{A}+\left(F_{x} \times r_{r_{x}}\right)}{J_{r_{\text {total }}}}, \\
\frac{d \dot{\omega}_{w}}{d t}=\frac{-T_{P}-\left(F_{x} \times r_{w_{x}}\right)}{J_{w_{\text {total }}}}
\end{array}
$$

where $J_{w_{\text {total }}}$ and $J_{r_{\text {total }}}$ are the total moment of inertias of wheel-roller respectively including electrical motors, all mechanical elements and connection components. These values for the wheel-roller are obtained as $17.86 \mathrm{kgm}^{2}$ and $47.2 \mathrm{kgm}^{2}$ by using a 3D modeling software, respectively. $T_{A}$ and $T_{P}$ are the applied torques by asynchronous motor and PMSM, respectively.

\section{Validation of the model}

Simulation scenarios, which are based on the torque request from PMSM, are generated. The torque request from the PMSM can be seen in Figure 12 for the first case. In the second case, the torque profile is same, but the maximum torque transmitted to the wheel is $852 \mathrm{~N}$ which is different than the first case $(500 \mathrm{~N})$. In these scenarios, for first and last 5 minutes, the system proceeds without traction. Initially, the asynchronous motor attached to the roller is controlled and adjusted to operate at synchronous frequency of $7.5 \mathrm{~Hz}$ for the first simulation scenario and $15 \mathrm{~Hz}$ for the second one, which is almost equal to the $15 \mathrm{~km} / \mathrm{h}$ and $30 \mathrm{~km} / \mathrm{h}$ translational velocity for the wheel in real case. According to the traction from PMSM, the asynchronous motor applies a counter torque which is proportional to the slip and given in Equation 1. Initial angular velocities for roller and wheel are calculated as $9.36 \mathrm{rad} / \mathrm{s}$ and $12.16 \mathrm{rad} / \mathrm{s}$ for the first case, and $18.73 \mathrm{rad} / \mathrm{s}$ and $24.33 \mathrm{rad} / \mathrm{s}$ for the second case, respectively.

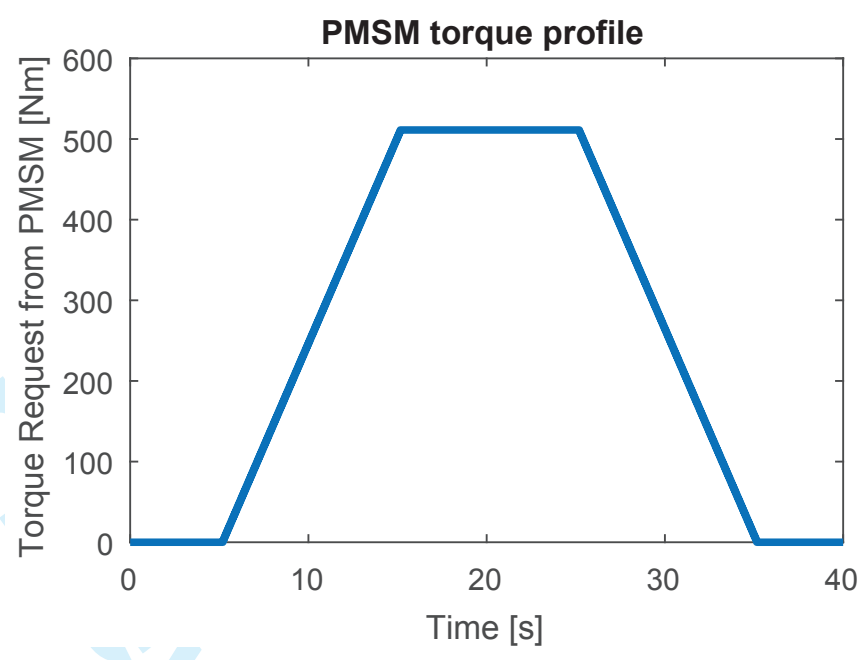

Figure 12. Torque request from PMSM

Simulation results and measurements for system states (i.e. angular velocities) can be seen in Figure 13 and Figure 14 for the first and second simulation scenarios, respectively. It is obvious that simulation results are in very good agreement with measurements for the first simulation scenario. However, when speed increases, because of the increasing dynamic effects due to modeling errors (especially due to unmodeled dynamics and characteristics of asynchronous motor), simulation results include more error than the first scenario during maximum traction. The difference between change in simulation and the change in measurement is approximately $11 \%$ for roller angular speed, and $9 \%$ for wheel angular speed. It can be seen from Figure 14 that the error is at an acceptable level. For the first and second simulation scenarios, the comparison of the results of the Fastsim algorithm with respect to measured creep force by using the torque transducer can be found in Figure 15. The error between creep force measurement and the results for the Fastsim in the second simulation scenario is approximately $7.3 \%$. While comparing with the errors in angular velocity measurements, error for creep force is smaller, but it should be noted that creep force is proportional with creep and the creep is dependent on the difference 
between velocities. Since the same trend is seen both for wheel and roller in the second simulation scenario, smaller error is obtained in the creep force comparison. Results for the Fastsim are obtained by using a 20x20 discretization.

\section{Unscented Kalman filter and model based estimation results}

The details of Unscented Kalman filter (i.e. UKF) for state and parameter estimation with some examples are previously reported $^{33-36}$ and the algorithm for UKF can be found in Appendix B. Furthermore, a similar study for a roller-rig with a different configuration and model is presented by Zhao et al. ${ }^{2}$, in which the torsional dynamics and UKF are considered to estimate the adhesion conditions. Instead of the adhesion conditions, the focus is to estimate the rolling radius of the wheel in this work. Considering the continuous time nonlinear system given in Equation 10, equations of the system can be written in the form ${ }^{37}$

$$
\begin{aligned}
\dot{x}(t) & =f(x(t), u(t), \theta)+q(t) \\
y(t) & =h(x(t), \theta)+r(t)
\end{aligned}
$$

where $x(t) \in \mathbb{R}^{n}$ is the system state vector, $u(t) \in \mathbb{R}$ is the input (in this case torque request from PMSM), $\theta$ is the parameter (i.e. rolling radius of the wheel), $y(t) \in \mathbb{R}^{m}$ is the measurement vector (i.e. system output), $q(t) \sim N(0, Q(t))$ and $r(t) \sim N(0, R(t))$ are process and measurement noises, respectively. In this study, the system is considered as discretized continous system and can be expressed by

$$
\begin{aligned}
& x_{k}=f_{d}\left(x_{k-1}, u_{k-1}, \theta_{k-1}, k-1\right)+q_{k-1} \\
& y_{k}=h\left(x_{k}, \theta_{k-1}, k-1\right)+r_{k},
\end{aligned}
$$

where $x_{k}=x(k T)$, the subscript $k$ represents discrete time, and $T$ is the sampling period. The function $f_{d}$ does not require an explicit formula, but a numerical integration is needed for calculation. In this study, fourth order RungeKutta numerical integration method with $0.25 \mathrm{~ms}$ time step is used. State vector can be given as

$$
\hat{x}=\left[\begin{array}{lll}
\hat{\omega}_{w} & \hat{\omega}_{r} & \hat{r}_{w_{x}}
\end{array}\right] .
$$

Output vector (i.e. measurement vector) can be given as

$$
y=\left[\begin{array}{ll}
\omega_{w} & \omega_{r}
\end{array}\right] .
$$

In order to deal with nonlinear dynamic systems, a linearisation method is required. In Extended Kalman Filter (i.e. EKF) linearisation is achieved by considering a linearisation of the dynamic model around the operating point. Another method for linearisation is to do a statistical linearisation. ${ }^{36}$ Instead of a dynamic model linearisation, a statistical linearisation is considered in UKF. Local linearisation considered in EKF introduces large errors in an highly nonlinear dynamic systems. ${ }^{35}$ In UKF, a set of sample points are initially created based on an initial error covariance (i.e. by using $P_{0}$ in Equation 15a) and these sample points are propagated through the nonlinear dynamic model. This statistical linearisation operation increases the accuracy of the filter and eliminates the need for a Jacobian
Matrix which is very difficult to obtain for highly nonlinear dynamic system. It should be noted that errors are assumed to be Gaussian Random Variables (i.e. GRV) in these deterministic filters (e.g. EKF, UKF).

First step in UKF is unscented transformation (i.e. UT) which is a deterministic sampling approach. Based on the initial estimate of states (in this case include parameter estimate as well) and covariance $P_{0}$, a set of samples are created. These sample points are called sigma points ${ }^{35}$. It can be seen in Figure 1 that sigma points corresponds to the dynamic models with different states (i.e. angular velocities) and parameter (i.e. rolling radius) in this study. These sigma points are propagated through mathematical models and state estimates for each sigma point are obtained in the form as given in Equation 13. Next step is to calculate a weighted mean for these sigma points and a priori error covariance (by considering this mean and the model noise matrix $-Q$ ) is obtained. This part is called time update for UKF. Then, aim is to obtain each measurement estimate for sigma points based on states. If there is a function which connects measurements and states, each sigma point should be also propagated through this function. In this study, there is no such a function since states in state vector and measurement vector is same. However, it should be noted that angular velocities (i.e. states) in Equation 13 and 14 are not same. Angular velocities given in Equation 13 represents states obtained by using mathematical models and angular velocities given in Equation 14 are the measurements taken from the physical system (e.g. railway vehicle, test stand etc.) by using sensory data.

Second step is to form a posteriori state estimate by using the mean of estimated measurements (in this case estimated measurements are $\hat{y}=\left[\begin{array}{ll}\hat{\omega}_{w} & \hat{\omega}_{r}\end{array}\right]$ ) and sensory measurements taken from the physical system. After calculating cross covariances between estimated measurements (by considering measurement noise matrix $R$ ), estimated states and mean of estimated measurements, a Kalman gain is obtained. Then, this gain is used to calculate a posteriori state estimate by using the priori estimated measurements and the measurements from the physical system. Last step is to update error covariance by using Kalman gain and cross covariance for estimated measurements. Shortly, the purpose is to minimize error for estimated states by considering measurements from the physical system.

Two estimation cases for each simulation scenario are defined. In the first case, the rolling radius of the wheel is initially assumed (i.e. estimated) as $320 \mathrm{~mm}$, whereas the measured rolling radius is approximately $348.2 \mathrm{~mm}$. This case is the underestimation case. In the second case, the rolling radius of the wheel is initially assumed (i.e. estimated) as $400 \mathrm{~mm}$ and this case is the overestimation case. These two cases are realized for the $15 \mathrm{~km} / \mathrm{h}$ and 30 $\mathrm{km} / \mathrm{h}$ translational velocities. Initial selection of the states are same as the ones indicated in model validation section. However, in the contact model, only a circular contact (2.7 $\mathrm{mm}$ radius) without shape correction is considered. This situation also increases uncertainty of the mathematical model and it is useful to test robustness of the estimator. In order to find a reference magnitude for the radius of the contact patch, the methods presented in the contact analysis 


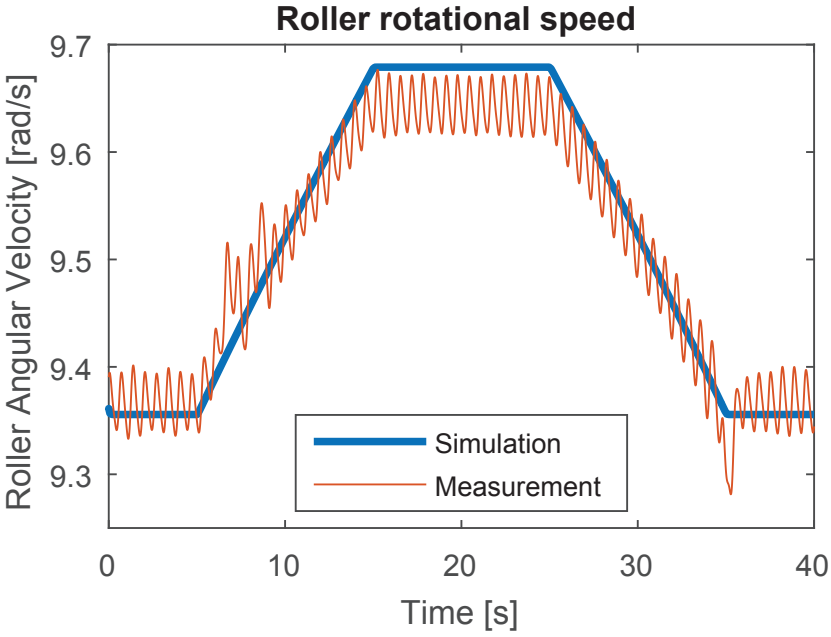

(a)

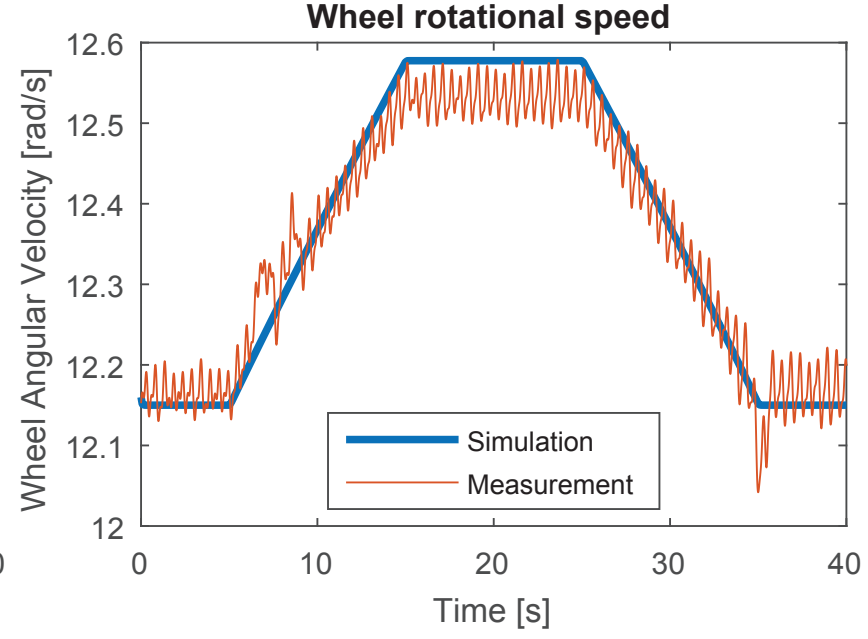

(b)

Figure 13. First simulation scenario $(15 \mathrm{~km} / \mathrm{h})$-comparison of simulation and measurements

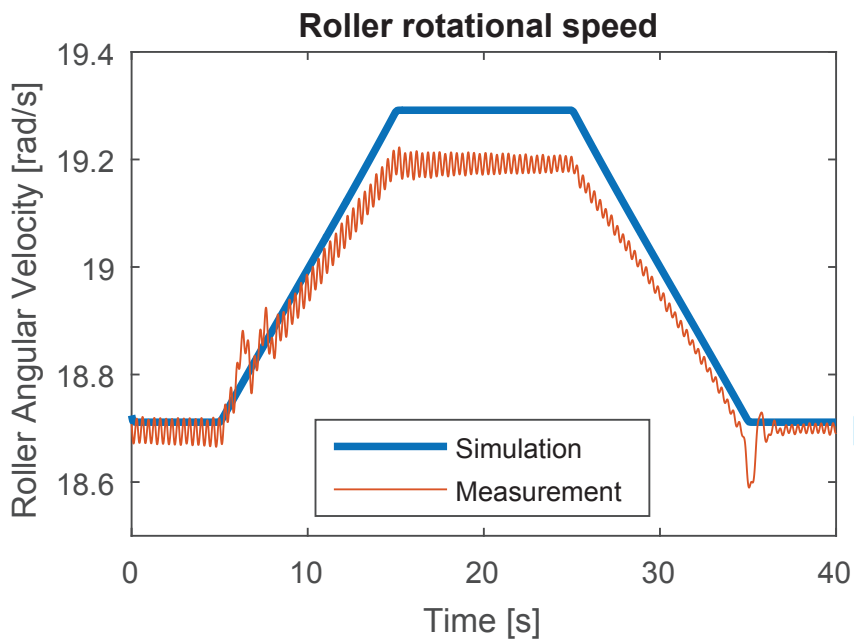

(a)

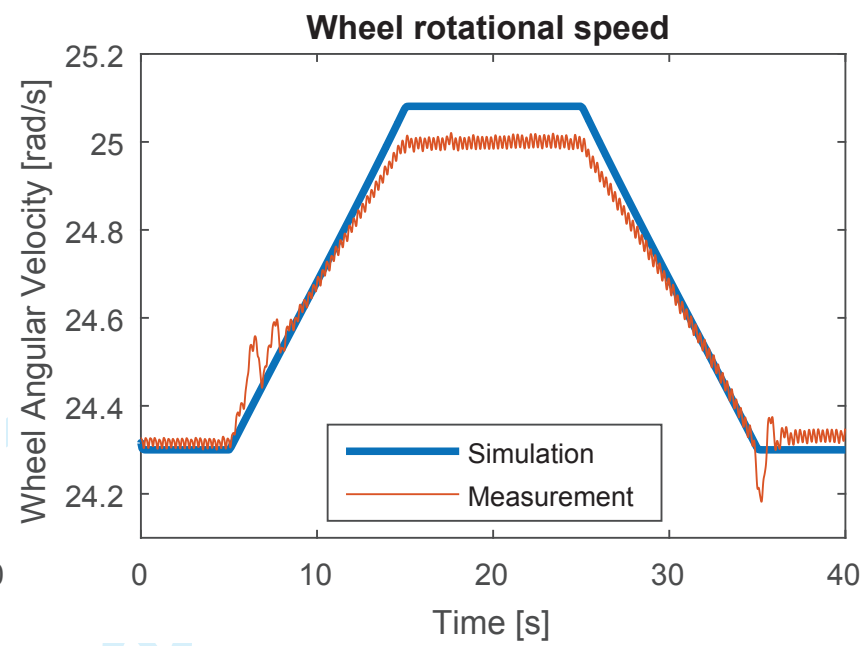

(b)

Figure 14. Second simulation scenario $(30 \mathrm{~km} / \mathrm{h})$-comparison of simulation and measurements

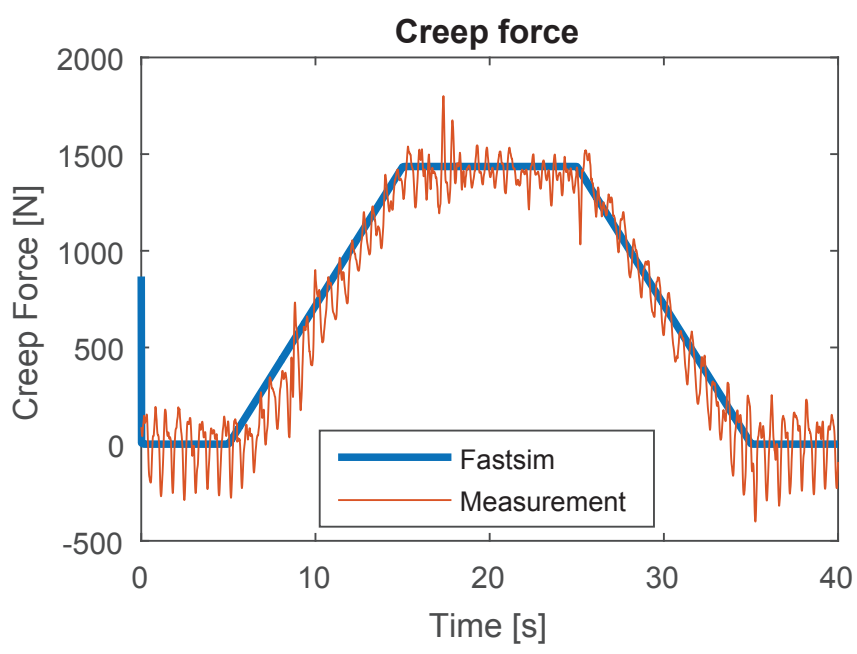

(a) First case $(15 \mathrm{~km} / \mathrm{h})$

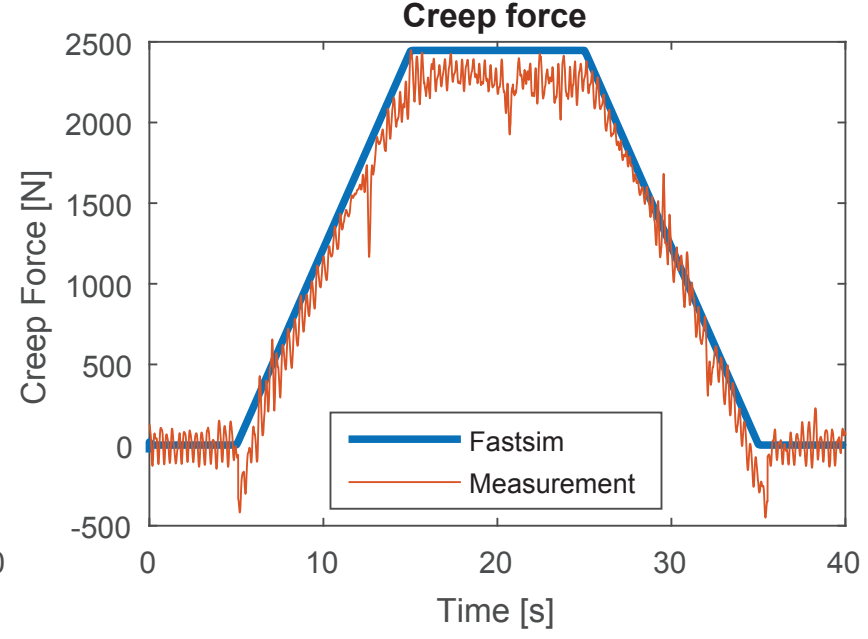

(b) Second case $(30 \mathrm{~km} / \mathrm{h})$

Figure 15. Comparison of creep forces between simulation (Fastsim) and measurement

section are taken into account. Furthermore, the filter of sigma points, $\kappa$ the secondary scaling parameter, and $\beta$ parameters $\alpha$ which is used to determine the distribution a non-negative weight incorporating the prior knowledge 
of the state distribution are taken 1,0 and 2, respectively. Filter parameter $\alpha$ is used to determine spread of sigma points around state estimate $\hat{x}$ and takes values between $10^{-4} \leq \alpha \leq 1$. Here, it is chosen as 1 so that the maximum exploration ability of UKF is used. $\kappa$ is the secondary scaling parameter and optimal value for this parameters is $3-L$ where $L$ is the number of states. ${ }^{36} \beta$ is used to incorporate prior knowledge of the distribution and for Gaussian distributions $\beta=2 .{ }^{34}$ Initial covariance matrix, process and measurement noise matrices are same for all conditions and given as

$$
\begin{aligned}
P_{0} & =\left[10^{-1}, 10^{-1}, 10^{-3}\right], \\
Q & =\left[10^{-5}, 10^{-5}, 10^{-5}\right], \\
R & =\left[10^{-5}, 10^{-5}\right] .
\end{aligned}
$$

There are seven parameters which can be selected for UKF. These are $\hat{x}_{0}$ (i.e. initial state estimation), $P_{0}, Q, R$, and UT parameters $\alpha, \kappa, \beta$. Selection of parameters used in UT (i.e. $\alpha, \kappa, \beta$.) are described by referring to the literature in the previous paragraph. It is stated by Han et al. ${ }^{38}$ that $\hat{x}_{0}$ and $P_{0}$ have asymptotically negligible influence on estimation results since UKF updates both of them as time proceeds. Nevertheless, $Q$ and $R$ influence UKF significantly. If they are chosen too small, estimated states will probably tighten, whereas if they are chosen too large, UKF may diverge. ${ }^{38}$ In this study, especially in order to tighten estimated states, $Q$ and $R$ are chose relatively small and their magnitudes are determined after extensive simulations. It is observed that relatively large value in $Q$ and $R$ (e.g. $10^{-1}$ ) causes divergence in UKF especially for the estimated parameter. It will be more appropriate to consider UKF versions with adaptive tuning mechanisms (e.g. the method which is presented by Han et al. ${ }^{38}$ ), if it is difficult to determine model and measurement noise covariance matrices $Q$ and $R$ by using extensive simulations.

Firstly, the effect of UKF on system states (i.e. angular velocities) are presented and can be found in Figures 16 and 17. It can be seen from Figures 16 and 17 that UKF provides better estimates of the angular velocities with respect to the cases presented in Figure 13 and 14 when a simulation model is considered only.

Estimation results for the first scenario $(15 \mathrm{~km} / \mathrm{h}$ translational velocity) are given in Figures 18 and 19. It is obvious from Figure 18 that UKF provides very fast convergence for underestimation case. Additionally, result of the overestimation scenario can be seen in Figure 19. Same conclusion can also be made for the overestimation case given in Figure 19.

Figures 20 and 21 represent the results of the underestimation and overestimation cases for the second estimation scenario (30 km/ $h$ translational velocity). Unlike the first estimation scenario, deteriorating effect of the higher speed due to modeling errors causes a little bit higher parameter estimation error for underestimation case in comparison with the first scenario. However, parameter estimation error for each case is at an acceptable level. Figure 20 reveals that UKF also provides very fast convergence in the second simulation scenario. Additionally, result of the overestimation case for second scenario is seen in Figure 21.
Table 1. Root mean squared errors for estimation scenarios

\begin{tabular}{|c|c|c|}
\cline { 2 - 3 } \multicolumn{1}{c|}{} & $\begin{array}{c}\text { First Estimation } \\
\text { Scenario } \\
(15 \mathrm{~km} / \mathrm{h})\end{array}$ & $\begin{array}{c}\text { Second Estimation } \\
\text { Scenario }\end{array}$ \\
\hline Underestimation & $0.6060 \mathrm{~mm}$ & $(30 \mathrm{~km} / \mathrm{h})$ \\
& $(\approx 0.17 \%)$ & $(\approx 0631 \mathrm{~mm}$ \\
& $0.8233 \mathrm{~mm}$ & $1.0139 \mathrm{~mm}$ \\
\hline Overestimation & $(\approx 0.24 \%)$ & $(\approx 0.29 \%)$ \\
\hline
\end{tabular}

Same conclusion can also be made for the overestimation case given in Figure 21.

For such estimators, a visual inspection of the results can be deceptive. Therefore, a different performance measure from the visual inspection should be considered. The performance measures for the family of Kalman filters are provided by Haug A. J. ${ }^{39}$. In this study, root mean squared error is used as the performance measure. Root mean squared error can be given as

$$
e_{R M S}=\left[\frac{1}{M} \sum_{i=0}^{M}\left(\theta_{i}-\hat{\theta}_{i}\right)^{2}\right]^{\frac{1}{2}}
$$

where $\theta$ represents the parameter (i.e. $r_{w_{x}}$ ) and $M$ represents the total number of sampled points. Root mean squared errors for the defined estimation scenarios can be found in Table 1. From the quantities of the root mean squared errors with respect to wheel radius $(348.2 \mathrm{~mm})$, it can be concluded that the estimation of the rolling radius is successful by using UKF and the largest amount of the root mean squared error is due to improper selection of the initial estimates which is about $50 \mathrm{~mm}$ over the nominal rolling radius for overestimation case and $30 \mathrm{~mm}$ under the nominal rolling radius for underestimation case.

Another issue, which should be mentioned in estimation and simulation scenarios, is the high frequency noise in the measurements. These high frequency vibrations are caused by two factors. The most influential factor is the mechanical imperfections of the test stand. The main frequency component of this periodic noise is equal to the frequency of rotation of the roller. This can be noted in Figures 13 and 14 . When the angular velocity doubles from $15 \mathrm{~km} / \mathrm{h}$ to $30 \mathrm{~km} / \mathrm{h}$, the frequency of noise also doubles. The other factor for the noise in velocity measurements is the numerical derivation procedure to obtain angular velocities from encoders. Encoders provide angular positions in radians and then during measurements derivatives of the angular position signals are taken. It is a well-known fact that derivation operation amplifies the noise effect. It is not a difficult task to filter out the noise from measurements by using a low-pass filter or a moving average filter. However, deterministic type of Kalman filters such as EKF and UKF provide optimal state estimation when models and measurements include Gaussian noise. Even though the statistical characteristics of the noise in the measurements of this study are not investigated, in order to exploit this feature of family of Kalman filters, use of a low-pass or moving average filter is avoided in this study. This type of noise is not only specific to test stand but also it can be observed for vehicles in operation. 


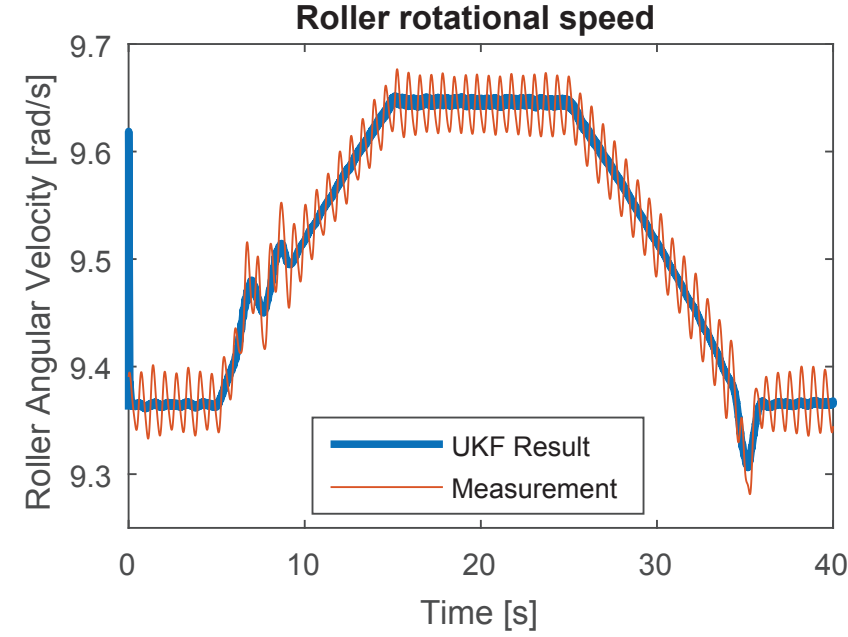

(a)

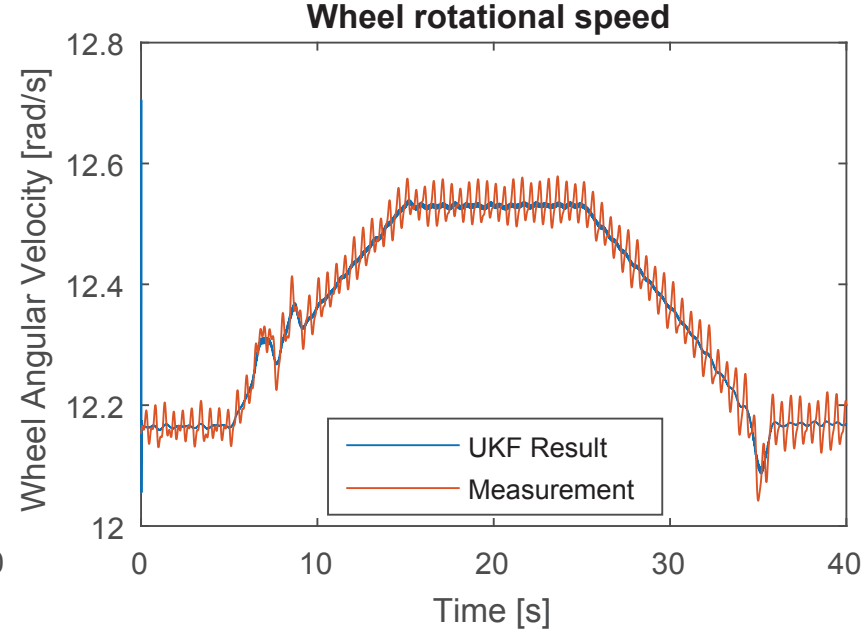

(b)

Figure 16. First simulation scenario $(15 \mathrm{~km} / \mathrm{h})$-comparison of UKF results and measurements

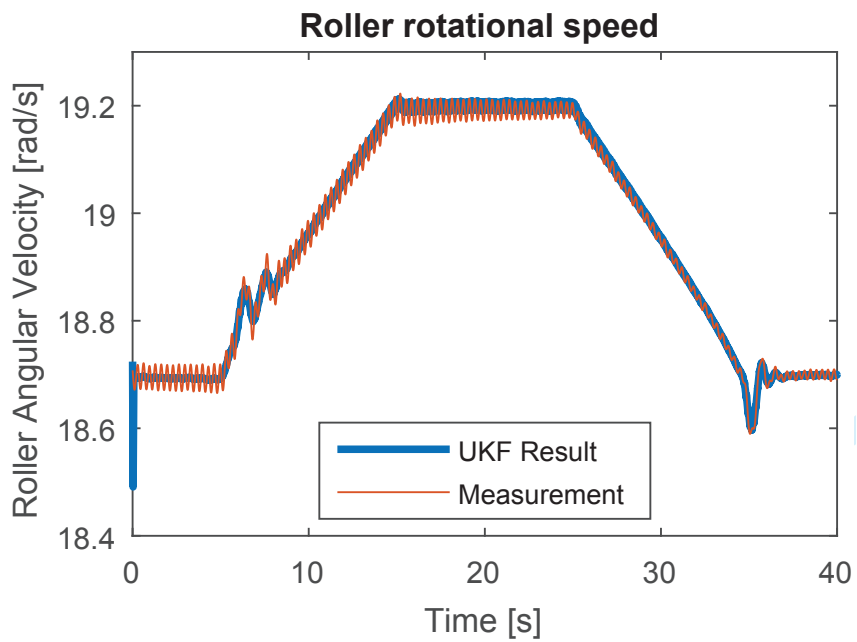

(a)

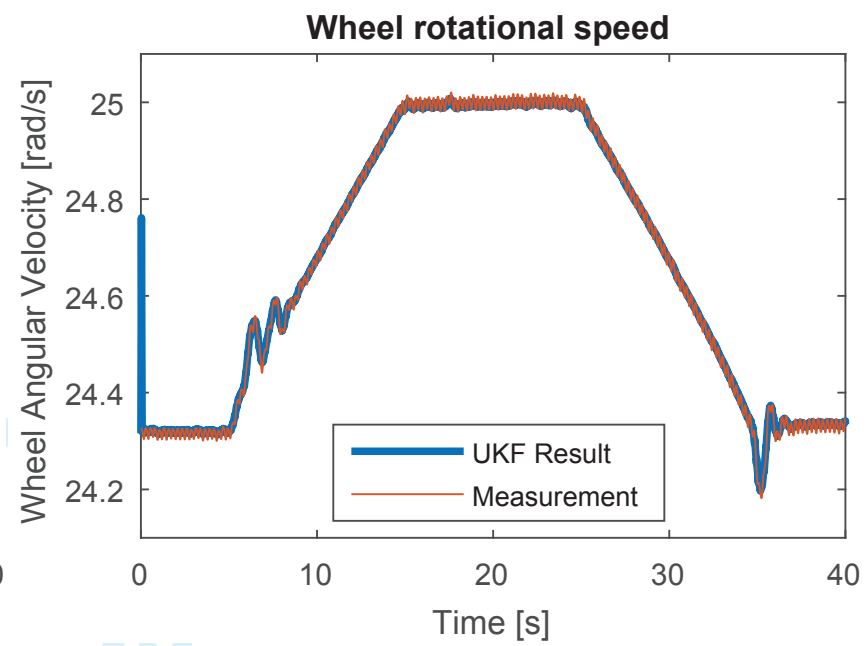

(b)

Figure 17. Second simulation scenario $(30 \mathrm{~km} / \mathrm{h})$-comparison of UKF results and measurements

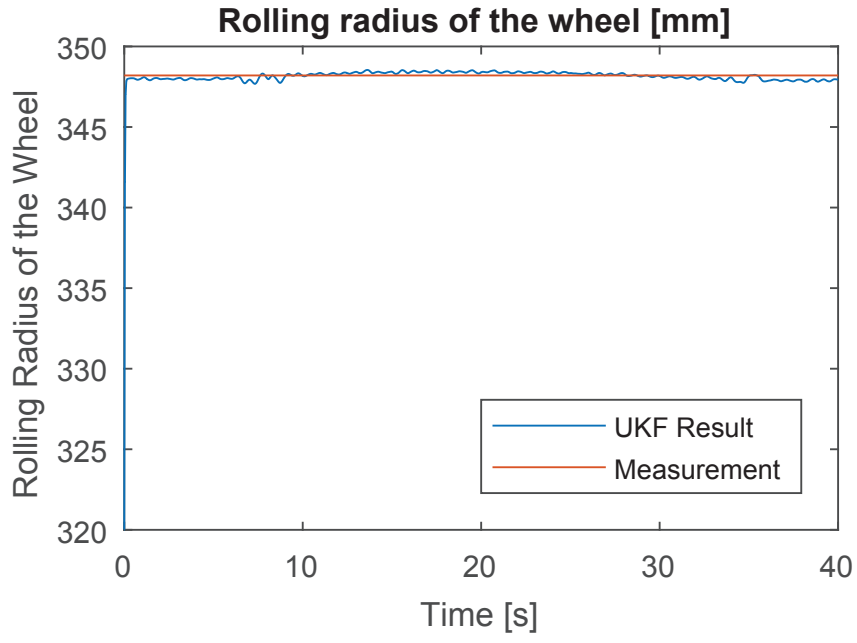

(a) Estimation result between $0-40$ seconds

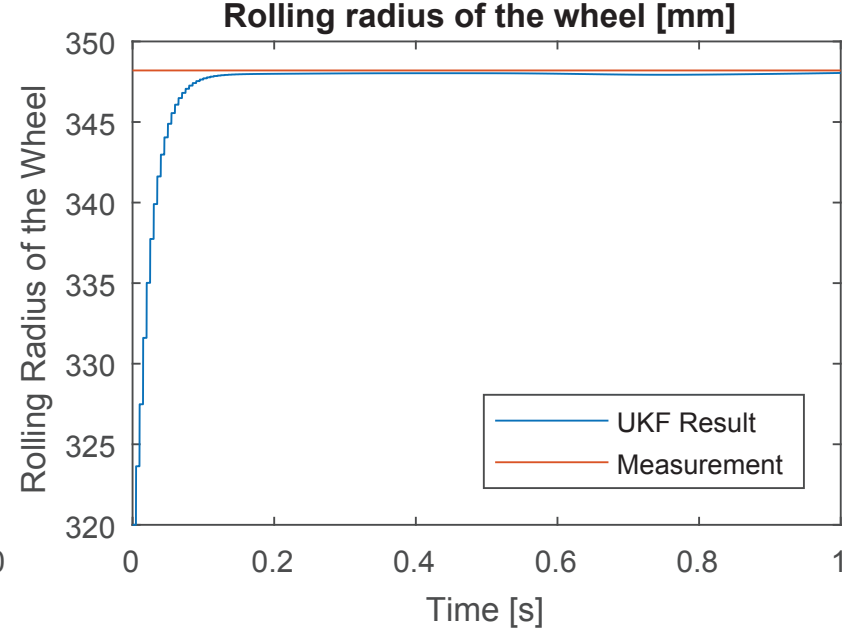

(b) Estimation result between $0-1$ second

Figure 18. First estimation scenario $(15 \mathrm{~km} / \mathrm{h}$ )-underestimation (initial estimate of $320 \mathrm{~mm}$ rolling radius)

\section{Discussion}

One may criticise the use of UKF for such a system instead of Kalman or Extended Kalman filter However, it can be seen from related equations that dynamic system is highly nonlinear in terms of system states (angular velocities). 


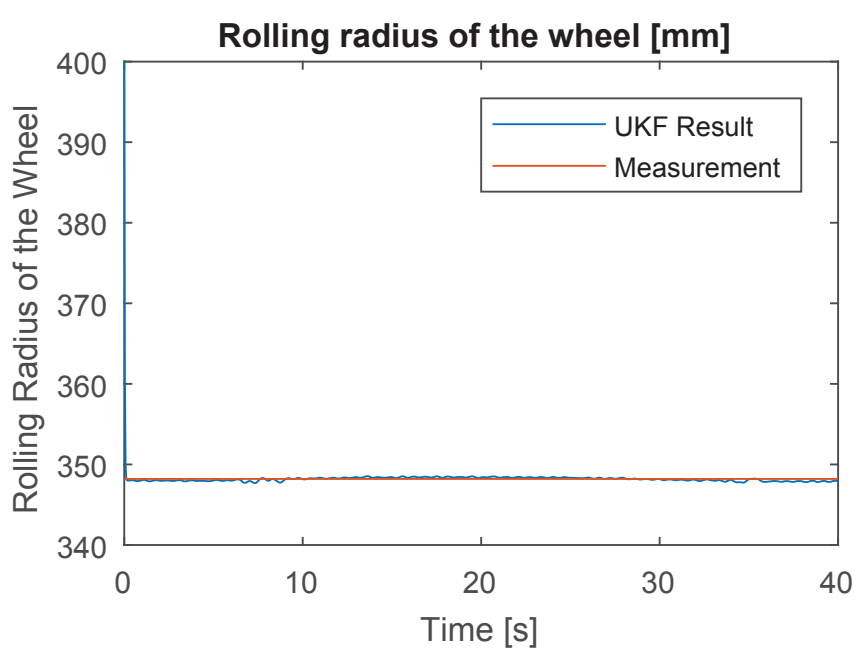

(a) Estimation result between 0-40 seconds

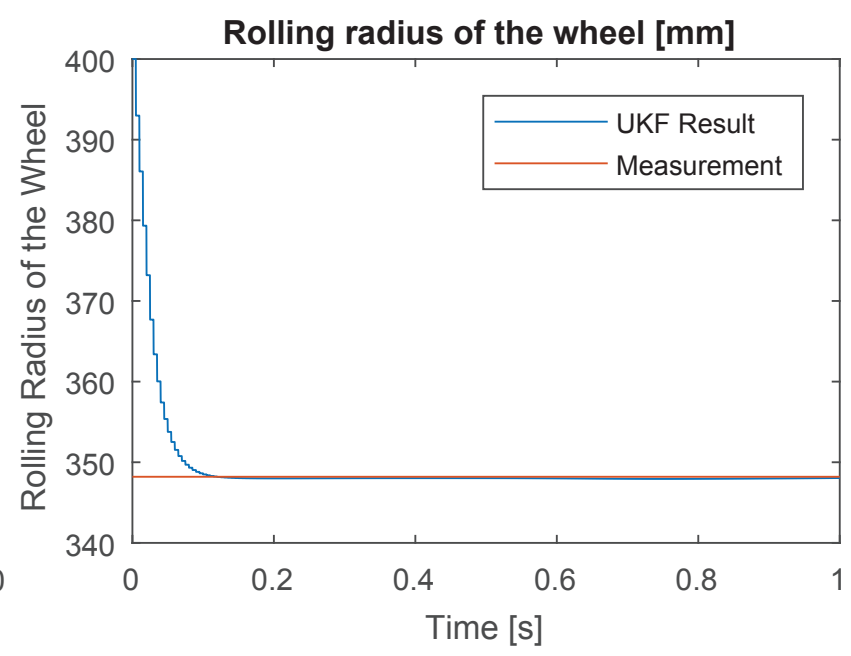

(b) Estimation result between $0-1$ second

Figure 19. First estimation scenario $(15 \mathrm{~km} / \mathrm{h}$ )-overestimation (initial estimate of $400 \mathrm{~mm}$ rolling radius)

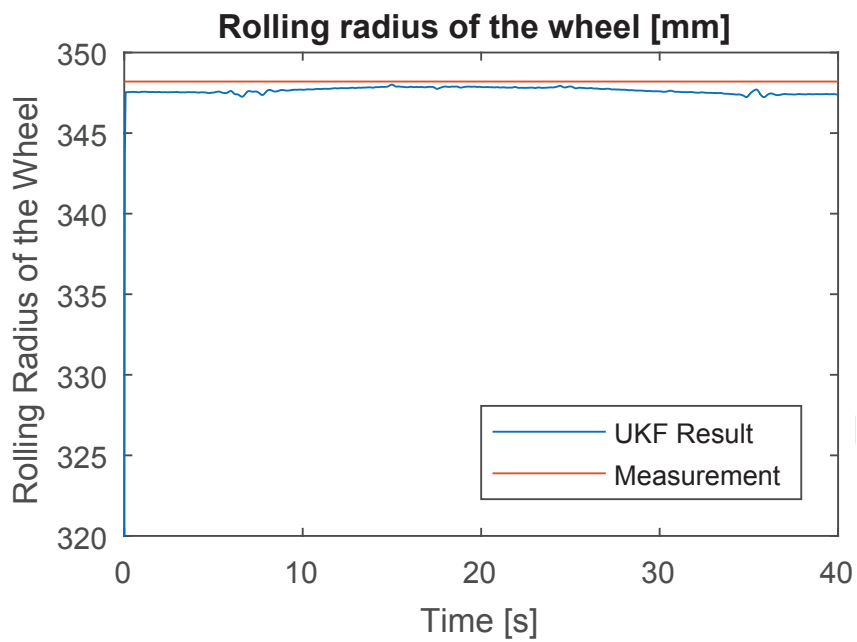

(a) Estimation result between $0-40$ seconds

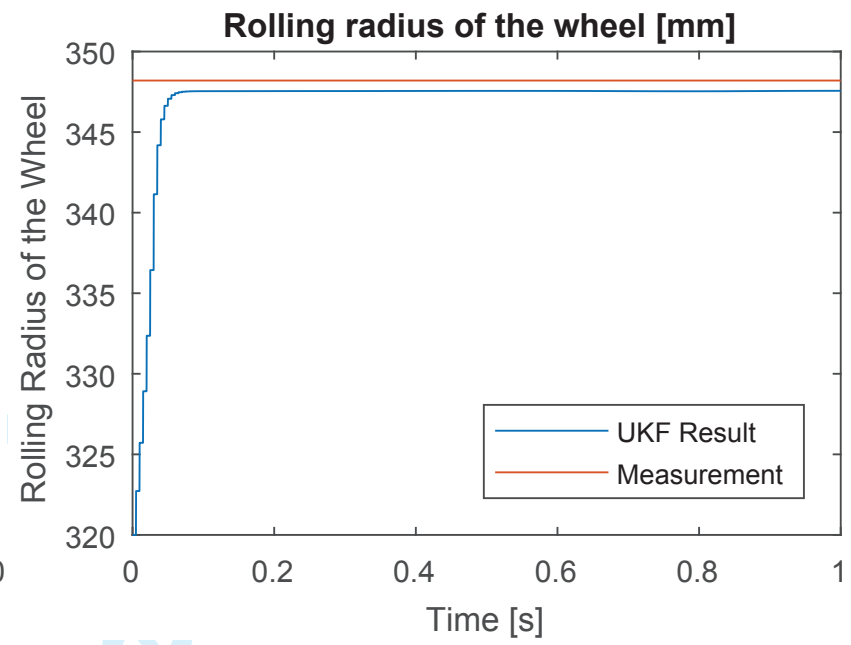

(b) Estimation result between $0-1$ second

Figure 20. Second estimation scenario $(30 \mathrm{~km} / \mathrm{h})$-underestimation (initial estimate of $320 \mathrm{~mm}$ rolling radius)

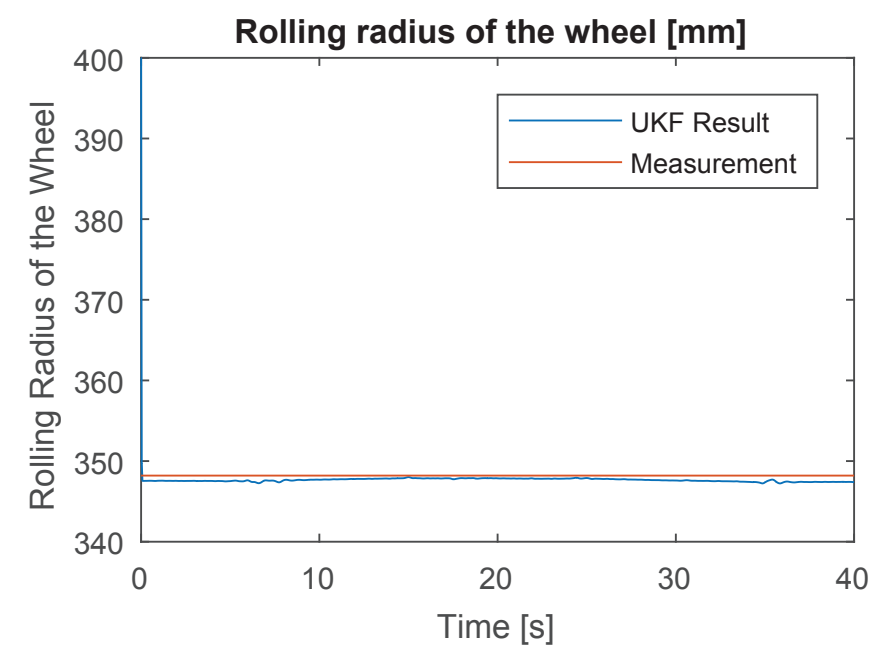

(a) Estimation result between 0-40 seconds

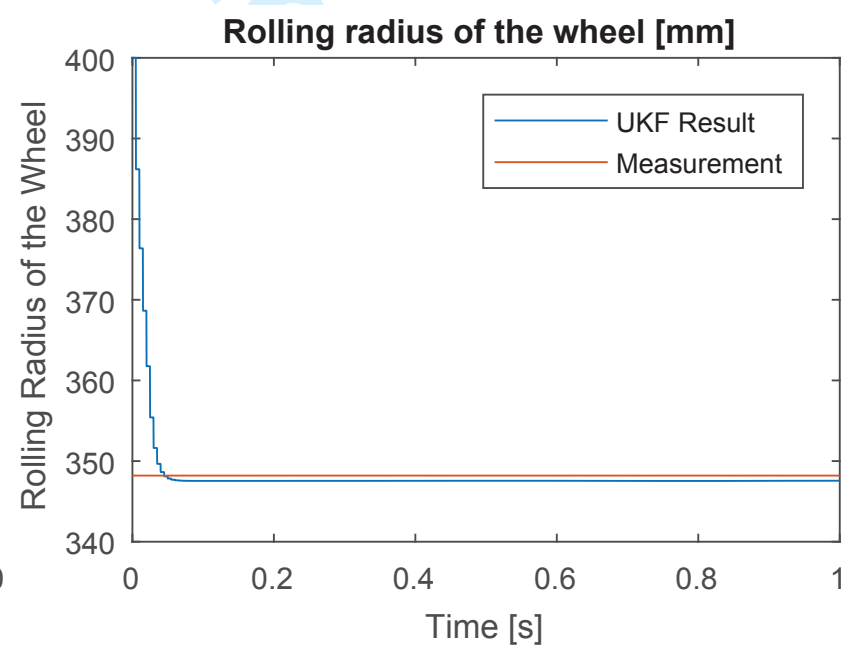

(b) Estimation result between $0-1$ second

Figure 21. Second estimation scenario $(30 \mathrm{~km} / \mathrm{h})$-overestimation (initial estimate of $400 \mathrm{~mm}$ rolling radius)

Due to this nonlinearity, the use of Kalman filter is not considerable. Extended Kalman filter requires the Jacobian matrix of the system. It can be seen in the model that obtaining a Jacobian is impossible due to the complex 
structure of the contact model. Therefore, UKF is the viable alternative for this application.

Secondly, one may question the use of a least squares estimator for parameter estimation instead of UKF. However, in the real case, as well as parameters, correct estimation of states is also essential. Especially in this case of application, estimating the parameter along with the states eliminates inaccuracies due to state estimation since the results of the models for asynchronous electric motor and tangential solution are highly dependent on the system states (i.e. angular velocities). A joint UKF is preferred for the state and parameter estimation so that both angular velocities and rolling radius are estimated accurately at one step. Furthermore, the accurate estimation of angular velocities for real vehicles is very important such that it leads to better slip control systems and longitudinal velocity calculation of vehicles.

Thirdly, for traction vehicles and locomotives, this estimation scheme can be used straightforwardly just by considering torsional dynamics of the wheel or wheelset (along with the traction motor), the contact model presented here, angular velocity measurement of the wheel or wheelset and translational velocity of the vehicle.

For wagons without traction and in steady rolling (i.e. without torque) of traction vehicles, this UKF based methodology can also be considered. However, different mathematical model directly related with wheel profile and continuous excitation should be taken into account In parameter estimation schemes there must be enough excitation (i.e. persistency of excitation) in the system to identify the parameter and here, the excitation is provided by electrical motor as torque which is not continuous in traction vehicles and do not exist in hauled stock. Fortunately, track irregularities can be a source of such excitation. Best suitable model is the vertical dynamic model (e.g. model given in Liang et al. ${ }^{5}$ ) of an railway vehicle system for such a purpose. By using UKF along with the vertical dynamic model of the vehicle and inertial measurement units, estimation scheme presented here can be used to monitor rolling radius of a wheel of a wheelset especially in the straight track sections with known vertical track irregularities. Drawbacks of such a scheme is the prior knowledge of vertical track irregularities and obtaining position of the wheel by using inertial measurement unit. However, when the amount of excitation (e.g. track irregularity) is known and the vertical position of the wheel is obtained by inertial measurement units, the magnitude of the inspected parameter (e.g. rolling radius) can be identified by using the model based estimation methodology presented in this study. This is out of scope of this study, but it is a challenging and interesting further research task to take. It has shown in Figures 18 and 19 that parameter estimation is achieved in steady rolling. However, this is due to the excitation provided by the asynchronous motor connected to the roller.

This study is carried out on a laterally fixed, independently rotating tram wheel. In practice, lateral track irregularities cause a lateral shift of the wheel or wheelset. Due to the curvilinear structure of the wheel, rolling radius changes with lateral shift of the wheelset. In order to make a comparison whether there is a wear and/or defect when the wheel is laterally shifted, knowledge of the lateral relative position of the wheel or wheelset with respect to the rail is required so that the amount of rolling radius difference for that lateral shift can be interpreted. Nevertheless, a low cost measurement system by using inertial measurement units without optical sensors do not exist for lateral relative position of wheel or wheelset with respect to the track. ${ }^{40}$ There are mathematical approaches to monitor wheel profile (i.e. conicity) with respect to a lateral relative wheelrail position. ${ }^{4}$ Nevertheless, such a study is limited with simulations and lack of experimental application. Combining the scheme presented here with the approach proposed by Charles et al. ${ }^{4}$, continuous profile estimation for different lateral shifts of the wheelset can be achieved in practice.

\section{Conclusion}

Monitoring of the rolling radius is important since severely worn wheels can cause safety problems for railway vehicles. The main goal of the current study is to propose a novel approach to simultaneously monitor the rolling radius of the wheel and angular velocities of the roller-wheel of the mentioned test stand. For this reason, firstly, a torsional dynamic analysis of a tram wheel test stand is presented. Then, it is concluded that the simulation model, which includes torque models of the electric motors and contact model, is sufficient to represent the behavior of the physical system. Then, the use of a joint UKF, which estimates states and parameter together, is proven to be effective tool for identifying rolling radius of the wheel. Estimation results show good performance of the joint UKF for rolling radius estimation for this test stand even with model uncertainties. Additionally, application of this approach for railway vehicles in practice is discussed and it is concluded that this scheme can be used by considering the mathematical model provided here for railway vehicles during traction.

\section{Acknowledgements}

Authors would like to thank prof. Ing. Jaroslav Novák, CSc. for his help and patience during the measurements from asynchronous motor.

\section{References}

1. Li C, Luo S, Cole C et al. An overview: modern techniques for railway vehicle on-board health monitoring systems. Vehicle System Dynamics 2017; 55(7): 1045-1070.

2. Zhao Y, Liang B and Iwnicki S. Friction coefficient estimation using an unscented kalman filter. Vehicle System Dynamics 2014; 52(sup1): 220-234.

3. Onat A, Voltr P and Lata M. A new friction condition identification approach for wheelrail interface. International Journal of Rail Transportation 2016; : 1-18DOI:10.1080/ 23248378.2016 .1253511$.

4. Charles G, Goodall R and Dixon R. A least mean squared approach to wheel-rail profile estimation. In Railway Condition Monitoring, 2008 4th IET International Conference on. pp. 16. DOI:10.1049/ic:20080346.

5. Liang B, Iwnicki S, Zhao Y et al. Railway wheel-flat and rail surface defect modelling and analysis by time-frequency techniques. Vehicle System Dynamics 2013; 51(9): 1403-1421. 
6. Real J, Salvador P, Montalbán L et al. Determination of rail vertical profile through inertial methods. Proceedings of the Institution of Mechanical Engineers, Part F: Journal of Rail and Rapid Transit 2011; 225(1): 14-23.

7. Alemi A, Corman F and Lodewijks G. Condition monitoring approaches for the detection of railway wheel defects. Proceedings of the Institution of Mechanical Engineers, Part F : Journal of Rail and Rapid Transit 2016; : 0954409716656218.

8. Voltr P. Simulation of wheel-rail contact conditions on experimental equipment. Railway Transport and Logistics 2015; XI: 77-82.

9. Liu B and Bruni S. Analysis of wheel-roller contact and comparison with the wheel-rail case. Urban Rail Transit 2015; 1(4): 215-226.

10. Iwnicki S. Handbook of railway vehicle dynamics. CRC press, 2006.

11. Bosso N, Spiryagin M, Gugliotta A et al. Mechatronic modeling of real-time wheel-rail contact. Springer, 2013.

12. Voltr P, Čáp J and Lata M. New practical results about adhesion limites obtained from experimental stand testing. Scientific papers of the University of Pardubice Series B, Jan Perner Transport Faculty 15 (2009) 2009; .

13. Voltr P, Lata M and Černỳ O. Measuring of wheel-rail adhesion characteristics at a test stand. Engineering Mechanics 2012; : 181.

14. Voltr $P$ and Lata $M$. Transient wheel-rail adhesion characteristics under the cleaning effect of sliding. Vehicle System Dynamics 2015; 53(5): 605-618.

15. Cernỳ O, Šimánek J, Dolecek R et al. Testing of robust control characteristics for traction pmsm. PIERS2007 2007; : 107-111.

16. Doleček R, Novák $\mathrm{J}$ and Černỳ $\mathrm{O}$. Traction permanent magnet synchronous motor torque control with flux weakening. Radioengineering 2009; 18(4): 601-605.

17. Meymand SZ. State of the Art Roller Rig for Precise Evaluation of Wheel-Rail Contact Mechanics and Dynamics. PhD Thesis, Virginia Tech, 2016.

18. Wang W, Zhang H, Wang $\mathrm{H}$ et al. Study on the adhesion behavior of wheel/rail under oil, water and sanding conditions. Wear 2011; 271(9): 2693-2698.

19. Bosso N, Gugliotta A and Zampieri N. Strategies to simulate wheel-rail adhesion in degraded conditions using a roller-rig. Vehicle System Dynamics 2015; 53(5): 619-634.

20. Diao L, Zhao L, Jin Z et al. Taking traction control to task: High-adhesion-point tracking based on a disturbance observer in railway vehicles. IEEE Industrial Electronics Magazine 2017; 11(1): 51-62. DOI:10.1109/MIE.2016.2644699.

21. Trummer G, Buckley-Johnstone L, Voltr P et al. Wheel-rail creep force model for predicting water induced low adhesion phenomena. Tribology International 2017; 109: 409-415.

22. Meymand S and Ahmadian M. Design, development, and calibration of a force-moment measurement system for wheelrail contact mechanics in roller rigs. Measurement 2016; 81: 113-122.

23. Sen PC. Principles of electric machines and power electronics. John Wiley \& Sons, 2007.

24. Arnold M and Netter H. Wear profiles and the dynamical simulation of wheel-rail systems. In Progress in Industrial Mathematics at ECMI 96. Springer, 1997. pp. 77-84.

25. Schupp G, Weidemann C and Mauer L. Modelling the contact between wheel and rail within multibody system simulation. Vehicle System Dynamics 2004; 41(5): 349-364.
26. Heckmann A, Keck A, Kaiser I et al. The foundation of the dlr railwaydynamics library: the wheel-rail-contact. In Proceedings of the 10 th International Modelica Conference; March 10-12; 2014; Lund; Sweden. 096, Linköping University Electronic Press, pp. 465-475.

27. Hertz H. Über die berührung fester elastischer körper. Journal für die reine und angewandte Mathematik 1882; 92: 156-171.

28. Onat A, Voltr P and Lata M. Nonlinear wheel/rail contact geometry characteristics \& determination of hertzian contact. Scientific papers of the University of Pardubice (Series B) 2014; 19: 145-152.

29. Ozdemir Y and Voltr P. Contact between wheel and rotating rail on a roller rig. In 33th International Colloquium-Advanced Manufacturing and Repair Technologies in Vehicle Industry.

30. Piotrowski J and Kik W. A simplified model of wheel/rail contact mechanics for non-hertzian problems and its application in rail vehicle dynamic simulations. Vehicle System Dynamics 2008; 46(1-2): 27-48.

31. Polach O. Creep forces in simulations of traction vehicles running on adhesion limit. Wear 2005; 258(7): 992-1000.

32. Kalker J. A fast algorithm for the simplified theory of rolling contact. Vehicle system dynamics 1982; 11(1): 1-13.

33. Julier SJ, Uhlmann JK and Durrant-Whyte HF. A new approach for filtering nonlinear systems. In American Control Conference, Proceedings of the 1995, volume 3. IEEE, pp. 1628-1632.

34. Wan E and Van Der Merwe R. Chapter 7: The unscented kalman filter. Kalman Filtering and Neural Networks 2001; :221-280.

35. Van Der Merwe R. Sigma-point Kalman filters for probabilistic inference in dynamic state-space models. PhD Thesis, Oregon Health \& Science University, 2004.

36. Matzuka B, Aoi M, Attarian A et al. Nonlinear filtering methodologies for parameter estimation. Department of Mathematics, North Carolina State University 2012; 1.

37. Zheng $M$, Ikeda $K$ and Shimomura $T$. Estimation of continuous-time nonlinear systems by using the unscented kalman filter. In SICE Annual Conference 2010, Proceedings of. IEEE, pp. 2192-2195.

38. Han J, Song Q and He Y. Adaptive unscented kalman filter and its applications in nonlinear control. In Kalman Filter Recent Advances and Applications. InTech, 2009.

39. Haug AJ. Bayesian Estimation and Tracking: A Practical Guide. John Wiley \& Sons, 2012.

40. Weston P, Roberts C, Yeo G et al. Perspectives on railway track geometry condition monitoring from in-service railway vehicles. Vehicle System Dynamics 2015; 53(7): 1063-1091. 


\section{A. Kalker coefficients}

The polynomial equations for Kalker's Coefficients. Here $k_{1}, k_{2}, k_{3}, k_{4}$ are auxiliary variables.

$$
\begin{aligned}
k_{1} & =2.3464+1.5443 . v+7.9577 . v^{2}, \\
k_{2} & =0.961669-0.043513 . v+2.402357 . v^{2}, \\
k_{3} & =-0.0160185+0.0055475 . v-0.0741104 . v^{2}, \\
k_{4} & =0.10563+0.61285 . v-7.26904 . v^{2}, \\
c_{11} & =k_{1}+\frac{k_{2}}{\left(\frac{b}{a}\right)}+\frac{k_{3}}{\left(\frac{b}{a}\right)^{2}}+\frac{k_{4}}{\sqrt{\left(\frac{b}{a}\right)}} . \\
k_{1} & =2.34641-0.27993 . v+0.19763 . v^{2}, \\
k_{2} & =0.96167+0.52684 . v+1.22642 . v^{2}, \\
k_{3} & =-0.0160185-0.0126292 . v-0.0011272 . v^{2}, \\
k_{4} & =0.10563+0.78197 . v-1.12348 . v^{2}, \\
c_{22} & =k_{1}+\frac{k_{2}}{\left(\frac{b}{a}\right)}+\frac{k_{3}}{\left(\frac{b}{a}\right)^{2}}+\frac{k_{4}}{\sqrt{\left(\frac{b}{a}\right)}} .
\end{aligned}
$$$$
k_{1}=0.29677+0.22524 . v+0.71899 . v^{2},
$$$$
k_{2}=1.01321+0.20407 . v-0.72375 . v^{2},
$$$$
k_{3}=0.0092415+0.0854262 . v+0.319940 . v^{2},
$$$$
k_{4}=\left(8.4835 \times 10^{-4}\right)-\left(3.211 \times 10^{-3}\right) \cdot v
$$$$
-\left(1.7484 \times 10^{-2}\right) \cdot v^{2}
$$$$
c_{23}=k_{1}+\frac{k_{2}}{\left(\frac{b}{a}\right)}+\frac{k_{3}}{\left(\frac{b}{a}\right)^{2}}+\frac{k_{4}}{\left(\frac{b}{a}\right)^{3}} .
$$$$
k_{1}=0.72795-1.00202 . v-0.32695 . v^{2},
$$$$
k_{2}=0.461755+1.002340 . v+0.081441 . v^{2} \text {, }
$$$$
k_{3}=0.023739-0.110640 . v+0.249008 \cdot v^{2} \text {, }
$$$$
k_{4}=-0.0012999+0.0063653 . v-0.0129114 . v^{2},
$$$$
c_{33}=k_{1}+\frac{k_{2}}{\left(\frac{b}{a}\right)}+\frac{k_{3}}{\left(\frac{b}{a}\right)^{2}}+\frac{k_{4}}{\left(\frac{b}{a}\right)^{3}} .
$$

$$
\begin{aligned}
& \text { 32: end function } \\
& \text { 33: function MeASUREMENT UPdate }\left(\chi_{k \mid k-1}^{*}, \mathbf{R}\right) \\
& \text { 34: } \quad \Upsilon_{k \mid k-1}^{*} \leftarrow h\left(\chi_{k \mid k-1}^{*}, u_{k-1}\right) \\
& \text { 35: } \quad \hat{\mathbf{y}}_{k}^{-} \leftarrow \sum_{i=0}^{2 L} \mathbf{W}_{i}^{(m)} \mathbf{\Upsilon}_{i, k \mid k-1}^{*} \\
& \mathbf{P}_{\tilde{y}_{k} \tilde{y}_{k}} \leftarrow \sum_{i=0}^{2 L} \mathbf{W}_{i}^{(c)}\left(\mathbf{\Upsilon}_{i, k \mid k-1}^{*}-\hat{\mathbf{y}}_{k}^{-}\right) \\
& \left(\Upsilon_{i, k \mid k-1}^{*}-\hat{\mathbf{y}}_{k}^{-}\right)^{T}+\mathbf{R} \\
& \mathbf{P}_{x_{k} y_{k}} \leftarrow \sum_{i=0}^{2 L} \mathbf{W}_{i}^{(c)}\left(\chi_{i, k \mid k-1}^{*}-\hat{\mathbf{x}}_{k}^{-}\right) \\
& \left(\Upsilon_{i, k \mid k-1}^{*}-\hat{\mathbf{y}}_{k}^{-}\right)^{T} \\
& \mathbf{K}_{k} \leftarrow \mathbf{P}_{x_{k} y_{k}} \mathbf{P}_{\tilde{y}_{k} \tilde{y}_{k}}^{-1} \\
& \hat{\mathbf{x}}_{k} \leftarrow \hat{\mathbf{x}}_{k}^{-}+\mathbf{K}_{k}\left(y_{k}-\hat{\mathbf{y}}_{k}^{-}\right) \\
& \mathbf{P}_{k} \leftarrow \mathbf{P}_{k}^{-}-\mathbf{K}_{k} \mathbf{P}_{\tilde{y}_{k} \tilde{y}_{k}} \mathbf{K}_{k}^{T} \\
& \text { end function }
\end{aligned}
$$

